\title{
Recent advances in modelling the ocean circulation and its effects on climate
}

\author{
David L T Anderson $\dagger$ and Jürgen Willebrand $\ddagger$ \\ $\dagger$ Department of Physics, Sub-Department of Atmospheric, Oceanic and Planetary Physics, Clarendon \\ Laboratory, Parks Road, Oxford OX1 3PU, UK \\ $\ddagger$ Institut für Meereskunde an der Universität Kiel, Düsternbrooker Weg, D-2300 Kiel, Federal Republic \\ of Germany
}

\begin{abstract}
The ocean plays a central role for the dynamics of the climate system. It can simultaneously act to damp certain components of the man-made climate changes, while amplifying others. At the same time, it is responsible for many aspects of climate variability which occur naturally (i.e. independent of man's activities). In the last decade there has been a considerable improvement in the skill with which the ocean can be modelled. There also have been advances in observing and monitoring certain aspects of the ocean circulation and thermal structure, although we are far short of a global ocean observing system.

The physical basis for ocean modelling is outlined. While three-dimensional models based on the Navier-Stokes equations are now well developed, the parametrization of unresolved motions and the specification of appropriate boundary conditions still cause many difficulties. The wind-driven circulation is now reasonably well understood. Increasing computer power has permitted an explicit inclusion of mesoscale eddies which are particularly crucial for the dynamics of western boundary currents such as the Gulf Stream. An area of considerable recent progress is the interaction of the tropical oceans with the atmosphere which leads to short-term climate fluctuations known as ENSO (El Niño-Southern Oscillation). On time scales of several months some rudimentary forecasts have been achieved although it is not yet clear how precisely the climate fluctuations associated with ENSO may be predicted.

Considerable uncertainties remain about climate variations on decadal and longer time scales. Due to differences in the way heat and fresh water are exchanged with the atmosphere, the thermohaline circulation can have more than one stable equilibrium state. Small changes in surface salinity can disrupt the thermohaline circulation and cause transitions to different climatic states within a few decades (so-called halocline catastrophe).
\end{abstract}

This review was received in its present form in June 1991. 


\section{Contents}

1. Introduction

2. Numerical circulation models

2.1. Governing equations

2.2. Surface boundary conditions

2.3. Parametrization of subgrid-scale processes

2.4. Numerical implementation

3. Observing the ocean circulation

3.1. Instruments and techniques

3.2. The use of data with models

4. Wind-driven circulation and the role of eddies

5. Short-term climate variability

5.1. A description of ENSO

5.2. Modelling ENSO

5.3. Predicting ENSO

6. Thermohaline circulation and long-term variability 29

$\begin{array}{ll}\text { 7. Concluding remarks } & 33\end{array}$ Acknowledgments $\quad 34$

References 


\section{Introduction}

The ocean with a heat capacity approximately 1000 times that of the atmosphere can store large quantities of heat relative to those needed to alter atmospheric balances. Not all of that massive heat reservoir is readily available, however, as pathways into the deep ocean where most of the heat capacity resides are limited. The associated timescales range from less than one year for the surface layers to about 1000 years for the deep ocean.

The ocean is a moving fluid, and besides storing heat it also transports large amounts of heat. That transport can only indirectly be inferred from observations, and present estimates suffer from considerable uncertainties. The maximum transport reaches 1-2 $\mathrm{PW}\left(1 \mathrm{PW}=10^{15} \mathrm{~W}\right)$ and is as important as the atmospheric transport, the oceanic contribution being greatest at lower latitudes while the atmospheric transport dominates at higher latitudes. In the annual average, heat is gained in the tropics and carried poleward where it is released to the atmosphere, thus contributing to a reduction of the pole-to-equator temperature gradient. A remarkable exception is the South Atlantic where apparently around $0.5 \mathrm{PW}$ are transported equatorward, i.e. up-gradient, into the North Atlantic.

Both storage and transport of heat are important in determining how the climate will adjust to the increase in radiatively active trace gases, such as carbon dioxide, methane and chlorofluorocarbons in the atmosphere. The global temperature responds with a delay to the radiative forcing, the time scale being dependent on where and how fast the heat can penetrate into the ocean thermocline and so the amplitude and regional distribution of the expected warming signal is strongly affected by large-scale ocean currents. Furthermore, the ocean can absorb the gases and thus directly affect the atmospheric radiation balance. The ocean may have absorbed up to $50 \%$ of the extra $\mathrm{CO}_{2}$ released as a result of man's activities although some estimates suggest a somewhat lower figure (Tans et al 1990), implying a missing sink of carbon. Vertical motions and water mass formation at high latitudes play a crucial role in both the heat and gas uptake. A quantitative understanding of the oceanic circulation is therefore a necessary prerequisite for any prediction of climate change.

Three-dimensional numerical ocean general circulation models are well suited for this purpose. The field of ocean modelling has developed rapidly over the last two decades, since the pioneering work of Bryan (1969). For a long time, the main emphasis was on the mean state of the circulation, the strength, location and dynamical balances of ocean currents, and the distribution of temperature, salinity and other water mass characteristics. Although many problems remain, models are now reasonably capable of simulating many aspects of the observed mean state and its seasonal variation. More recently, changes in circulation patterns on interannual and longer time scales have attracted growing interest, in connection with possible climate changes.

The question of climate change due to man-made causes cannot be separated from the issue of natural variability in the coupled ocean-atmosphere system. From an observational point of view, the presence of fluctuations makes the detection of secular changes from data very difficult, and has so far prevented a clear identification of the expected global warming trend caused by greenhouse gases. From a modeller's point 
of view, it is obvious that the same dynamical principles apply to both anthropogenic and natural changes, and one cannot hope to understand the former without having understood the latter.

The last decade has seen a marked increase in observations of the tropical oceans and atmosphere, in understanding of tropical climate variability and in appreciation of the key role of the ocean in that climate variability. Climatic disruptions on interannual timescales are associated with ENSO (the El Niño Southern Oscillation), which is centred on the tropical Pacific but influences climate as far afield as India and the Sahel and on occasion possibly even Europe. It is not the sole mechanism causing climate variability in the tropics, however, and while, for example, most drought years over India are ENSO years, i.e. years when sea surface temperature (ssT) in the equatorial Pacific is anomalously warm there are droughts in non-ENSO years as well as ENSO years with no droughts. Climate variability is often associated with more than one phenomenon, making it difficult to isolate the relevant physics. The reader is referred to McCreary and Anderson (1991) for a review of modelling and theoretical work, to Enfield (1989) for a review of observed features of ENSO and to Philander (1990) for an account of equatorial dynamics, both atmospheric and oceanic, pertinent to ENSO.

Much less is known about climate variability on longer time scales. The record of global average temperature indicates a warming by $0.5^{\circ} \mathrm{C}$ since the beginning of this century, with considerable fluctuations on decadal time scales (Hansen and Lebedeff, 1988). Significant spatial differences also exist, as demonstrated by Parker et al (1988) who determined the principal component $\dagger$ of the variation in global sea surface temperature (ssT) during the last 90 years. Figure 1.1 illustrates a general North-South gradient in the third component of sst variability, with opposing signs in both hemispheres. The dominant time scale is several decades, with a temperature increase in the North Atlantic from 1920 to 1960 followed by a cooling in the two decades before the temperature rise in the 1980s. The origin of this variability which is correlated with rainfall in the Sahel zone is not well understood, but almost certainly involves the ocean circulation as the dominant time scale of atmospheric processes is only a few weeks.

The circulation in the ocean is driven by the wind and by thermohaline forcing (fluxes of heat and fresh water which cause density differences at the surface). Time scales of variability are months to years for the wind-driven response, and decades to centuries for the thermohaline circulation. However, due to strong nonlinearities the circulations resulting from both forcings interact, and the thermohaline circulation is hence not a consequence of thermohaline forcing alone. A principal difficulty when modelling long-term variations is that ocean models cannot be run alone, in isolation from the atmosphere. While the long time scales most likely result from ocean dynamics, the strong ocean-atmosphere coupling induces atmospheric variations which must also be modelled. Only recently has it become possible to run coupled models for several decades to explore temporal variations of the coupled system.

The purpose of this review is to acquaint the reader with the current state of ocean circulation models, their ability to model the present climate state and its variability,

† A data set representing space-time variability can be decomposed into a number of spatial patterns with corresponding temporal evolution. These patterns, called principal components or empirical orthogonal functions, are chosen to maximally reproduce the variance in the original data set. See Preisendorfer (1988) for a review of this and related methods. 

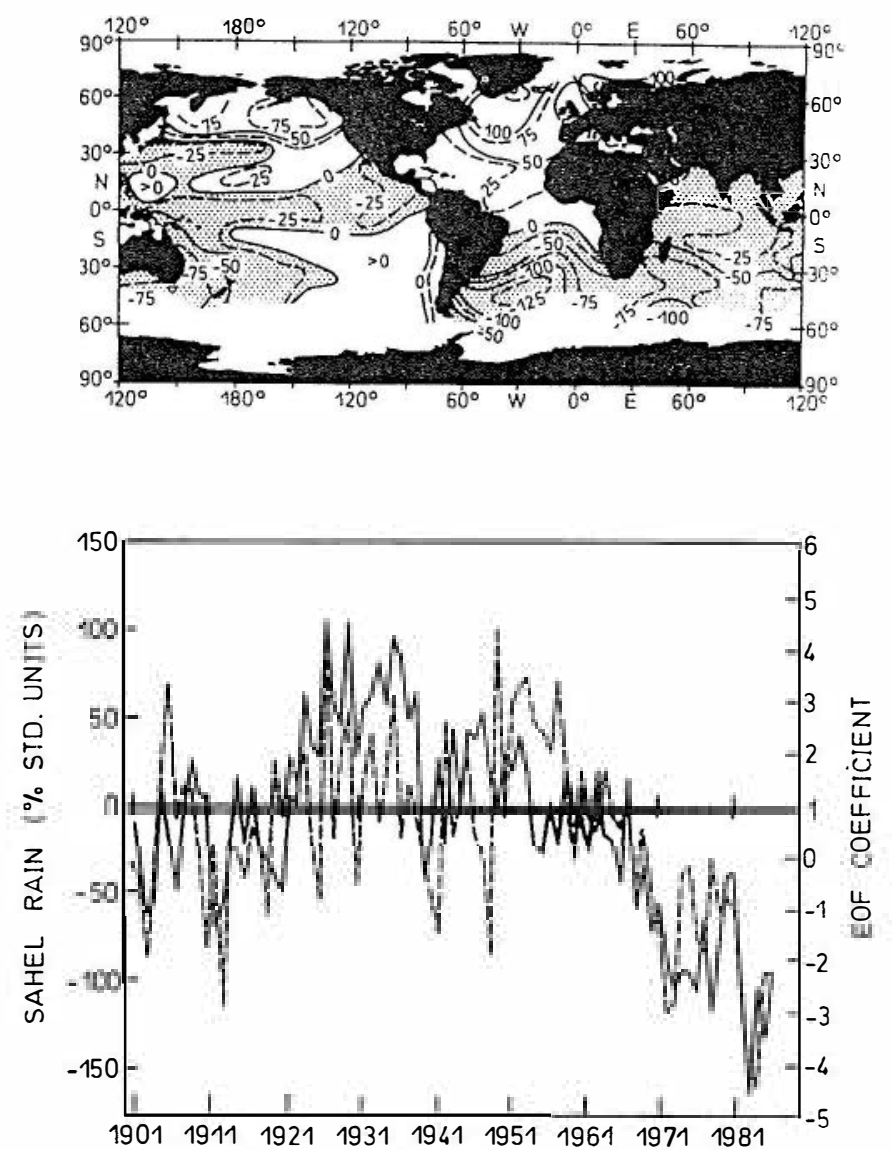

Figure 1.1. (a) Spatial pattern of the third empirical orthogonal function (EOF) component of worldwide sea surface temperature from 1901-80. (b) Amplitude time series of the EOF (full line), and annual rainfall in the Sahel zone (dashed). After Parker et al (1988).

and their major shortcomings and uncertainties. We will limit the discussion to threedimensional models of the physical system, and not address in detail models of chemical and biological processes which are necessary for understanding the ocean carbon cycle. Modelling of the carbon cycle is still at an early stage; the reader is referred to Bacastow and Maier-Reimer (1990) for a recent paper on this subject.

The paper is organized as follows. Section 2 will describe the basic structure of circulation models, and discuss various problems with their implementation. Section 3 gives a brief overview of the types of observational data in oceanography, and the ways in which the data are used. In section 4 some results from models of the wind-driven circulation are discussed, with particular emphasis on the dynamics of mesoscale eddies. Considerable progress has been made in understanding short-term variability associated with ENSO, and section 5 describes ocean-atmosphere interactions in the tropics as well as results from coupled ocean-atmosphere models for ENSO variability. In section 6 , models of the thermohaline circulation are described and some emerging ideas regarding long-term changes are given. 


\section{Numerical circulation models}

\subsection{Governing equations}

Ocean processes that are relevant to climate change occur mainly at large scales, ranging from less than $100 \mathrm{~km}$ (typical of intense boundary currents such as the Gulf Stream) to global, and at temporal scales ranging from a few months (exchange processes in the upper ocean) to over 1000 years (processes involving the deep circulation). In this scale range, oceanic motions are very nearly in geostrophic equilibrium, i.e. the horizontal pressure gradient is balanced by the Coriolis force, except for some processes near boundaries and the equator.

The principal mechanisms which govern the evolution of the oceanic state in response to forcing at the surface are (i) various waves, in particular planetary waves, also known as Rossby waves which provide the adjustment of the ocean mass field to wind forcing on time scales up to a few years, and (ii) the formation, advection and mixing of water mass properties which lead to changes in the temperature, salinity and density of the fluid in response to surface heat and fresh water fluxes. Ocean models for use in longer-term climate change problems must explicitly include those processes. However, other processes which span a much wider range of scales can also influence the large-scale circulation. Of particular importance are internal instabilities which draw energy both from the kinetic energy of the circulation (shear instability) and from its potential energy (baroclinic instability). The preferred scale of these instability processes is the Rossby deformation radius which depends on density stratification and latitude, and in mid-latitudes is $30-50 \mathrm{~km}$ in the ocean, in contrast to $1000 \mathrm{~km}$ for the corresponding scale in the atmosphere.

The equations governing oceanic motions are derived from basic conservation principles (Navier-Stokes equations, mass conservation, first law of thermodynamics). No single model can simultaneously resolve all the processes referred to above, and it is necessary to focus on a certain subset of processes and/or scales of motion. This is normally achieved by considering approximate (and often simplified) systems which are valid only for certain scales or for certain processes.

The most widely used basis for ocean circulation models is the primitive equation (PE) system. It is given by

$$
\begin{aligned}
& \frac{\mathrm{d} \boldsymbol{u}}{\mathrm{d} t}+2 \boldsymbol{\Omega} \times \boldsymbol{u}+\frac{1}{\rho_{0}} \nabla p=\boldsymbol{q} \\
& \frac{\partial p}{\partial z}+g \rho=0 \\
& \frac{\partial \omega}{\partial z}+\nabla \cdot \boldsymbol{u}=0 \\
& \frac{\mathrm{d} T}{\mathrm{~d} t}=q_{T} \\
& \frac{\mathrm{d} S}{\mathrm{~d} t}=q_{S} \\
& \rho=\rho(S, T, p) .
\end{aligned}
$$

The state variables $\boldsymbol{u}, \omega, p, \rho, T, S$ denote horizontal velocity, vertical velocity, pressure, density, potential temperature and salinity, respectively; $\rho_{0}$ is a constant refe rence 
density; $\boldsymbol{\nabla}$ is the horizontal gradient operator, $z$ is the vertical coordinate, $\Omega$ is the vertical component of the Earth's angular velocity, $g$ is gravity taken to be constant, and $d / d t$ is the material derivative t following the particle motion. The terms on the right-hand side of 2.1, 2.4 and 2.5 result from small-scale unresolved motions which will be discussed in section 2.3. The thermodynamic equation of state (2.6) is empirically known to better than $10^{-5}$ relative accuracy.

The system (2.1)-(2.6) is based on a number of approximations. The hydrostatic balance (2.2) is the most important consequence of the shallow-water approximation which is based on the small ratio of vertical to horizontal scales. The shallow-water approximation is normally well satisfied, but eliminates small-scale convective motions which must be included in parametrical form. The Boussinesq approximation neglects small density differences in the ocean except when gravity effects are important. As a consequence, continuity is approximated by the incompressibility condition (2.3), and acoustic waves with their fast propagation speed are eliminated from the system. For a fuller derivation of these equations see Gill (1982).

Besides the PE system, quasigeostrophic (QG) models have been widely used in the last decade. The QG equations can be derived from (2.1)-(2.6), under the additional assumptions that deviations from geostrophy in (2.1) are small, and that the lateral scales are much smaller than the radius of the earth so that the density surfaces are nearly horizontal. The derivation of the QG equations from (2.1)-(2.6) is a little technical and not included here: the reader is referred to Holland (1978) for a detailed description. The conceptual simplicity and computational economy of QG models (typically one order of magnitude faster than comparable PE models), make them excellent tools to study some principal aspects of wind-driven circulation in mid-latitudes. In particular, QG models have been run at high resolution to study instabilities and the turbulence cascade. They are however less well suited for realistic simulations as they do not include diabatic processes related to thermal forcing, and do not fully represent topographic effects.

Currently under development are models based on balanced equations (McWilliams et al 1990) which constitute an attempt to keep the economy and simplicity of the QG system without the corresponding loss of accuracy. Other approximations such as those made in reduced gravity models have been useful in simulating the response of the upper ocean to wind forcing, especially in the tropics. For a description of various model formulations in use see O'Brien (1986) or Anderson and Willebrand (1989).

\subsection{Surface boundary conditions}

At the ocean surface, the fluxes of momentum, heat and fresh water must be specified. The momentum flux through the sea surface occurs via wind-generated surface gravity waves. Its evaluation strictly requires a specification of the full wave spectrum but usually this is simplified and the normal component of the momentum flux tensor (wind stress $\tau$ ) is parametrized in terms of the wind velocity $U$ as

$$
\tau=\rho_{\mathrm{a}} C_{\mathrm{D}}|\boldsymbol{U}| \boldsymbol{U}
$$

where $\rho_{\mathrm{a}}$ is atmospheric density and $C_{\mathrm{D}}$ is an empirical drag coefficient which depends on atmospheric stability and other parameters. The accuracy of wind stress fields

$\dagger$ The material derivative $d / d t$ can be written as $\partial / \partial t+u \cdot \nabla+\omega \partial / \partial z$. The $d / d t$ terms in eqns (2.1), (2.4) and (2.5) are hence nonlinear and it is this nonlinearity which gives rise to many of the interesting phenomena in the ocean (and atmosphere). 
calculated using (2.7) is usually not better than $20-30 \%$ and regionally much worse, partly due to uncertainties in the drag coefficient and partly due to sparse wind observations. The momentum flux provides a one-sided coupling between ocean and atmosphere (the atmosphere forces the ocean but not vice versa). While the momentum flux from the atmosphere is an important aspect of the atmospheric circulation it is largely determined by the atmosphere in response to heating. By contrast the ocean largely responds to the momentum exchange and only indirectly influences it, through changes in SST resulting from advection and vertical mixing induced by the momentum flux.

The situation is very different for the fluxes of heat and fresh water. Empirical ('bulk') formulae relate sensible, latent and radiative heat flux and evaporation to ocean surface temperature and various atmospheric parameters, notably wind, air temperature, moisture, and cloudiness. (The familiar exchange of heat between two bodies at difference temperatures is called the sensible heat flux to distinguish it from the heat required to evaporate water called the latent heat flux.) The net heat flux $H_{T}$ and the net fresh water flux $H_{S}$ (from evaporation, precipitation, melting and freezing) can hence be expressed as

$$
\begin{aligned}
& H_{T}=H_{T}\left(T, a_{1}, a_{2}, \ldots\right) \\
& H_{S}=H_{S}\left(T, a_{1}, a_{2}, \ldots\right)
\end{aligned}
$$

where $T$ denotes the sea surface temperature (SST) and $a_{1}, a_{2} \ldots$ atmospheric variables (including radiation parameters and precipitation). Typical accuracies are $30-40 \mathrm{~W} \mathrm{~m}^{-2}$ for the observed annual mean net heat flux, and $20-50 \mathrm{~cm} \mathrm{y}^{-1}$ for fresh water flux. (For comparison, the increase in radiation as a consequence of $\mathrm{CO}_{2}$-doubling is around 3-4 $\mathrm{W} \mathrm{m}^{-2}$ !)

The dependence of (2.8) on both oceanic and atmospheric parameters provides a true coupling between ocean and atmosphere, i.e. the evolution of each system depends on the state of the other. Atmospheric models are frequently run in stand-alone mode using (2.8), with prescribed ocean surface temperature. This is appropriate as atmospheric time scales are generally much shorter than those in the ocean. For ocean models, however, a corresponding procedure (i.e. specifying the atmospheric parameters) is not adequate as it ignores the rapid atmospheric adjustment to ocean SST.

In early ocean models surface temperature and salinity were simply specified and the fluxes necessary to maintain those specified fields diagnosed a posteriori. However, these fluxes were not consistent with (2.8) implying that the atmosphere would not be in equilibrium with the ocean, as atmospheric adjustments to ocean changes had been ignored. Frequently used alternative formulations are

$$
\begin{aligned}
& H_{T}=\lambda_{T}\left(T^{*}-T\right) \\
& H_{S}=\lambda_{S}\left(S^{*}-S\right)
\end{aligned}
$$

which effectively restore surface temperature and salinity to prescribed values $T^{*}$ and $S^{*}$, with timescales related to the coefficients $\lambda_{T}^{-1}$ and $\lambda_{S}^{-1}$, respectively. Equations (2.9) constitute an atmospheric model, albeit a very simple one. Equation (2.9a) can be considered as a linearization of $(2.8 a)$, and both the virtual temperature $T^{*}$ and the coefficient $\lambda_{T}$ can in principle be calculated from atmospheric parameters. Typical local values of $\lambda_{T}$ would be around $50 \mathrm{~W} \mathrm{~m}^{-2} \mathrm{~K}^{-1}$. However, to account for the atmospheric response to SST, resulting in life times of a few months for SST anomalies at $1000 \mathrm{~km}$ scales, somewhat smaller values $\left(10-30 \mathrm{~W} \mathrm{~m}^{-2} \mathrm{~K}^{-1}\right)$ are normally used. In 
general $\lambda_{\tau}$ decreases for larger scales, down to $2 \mathrm{~W} \mathrm{~m}^{-2} \mathrm{~K}^{-1}$ for the global averages. The corresponding formulation $(2.9 b)$ for fresh water flux has however no physical basis as neither evaporation nor precipitation depend on surface salinity. The choice of flux conditions is particularly crucial for the study of long-term variability, as will be discussed further in section 6.

\subsection{Parametrization of subgrid-scale processes}

The state variables in (2.1)-(2.6) represent average over those spatial/temporal scales which are not explicitly resolved. The interaction with unresolved motions occurs via the forcing terms in (2.1), (2.4), (2.5), the divergences of the subgrid scale fluxes of momentum, heat and salt. Subgrid scale parametrization is perhaps the least satisfactory aspect of ocean models. The specifications of turbulent fluxes in terms of the mean fields and/or other known variables constitutes a formidable closure problem, which is aggravated by the broad range of scales and processes which are potentially relevant. Theoretical investigations have had rather limited success as reviewed by Holloway (1989). It is obvious that the magnitude of those fluxes depends on the scale over which the average is performed, and hence parametrization and resolution are very closely linked. Although lacking a strong justification, most models have used simple diffusive (Fickian) parametrizations, with the choice of the coefficients often dictated by numerical expediency rather than by physical arguments.

The amount of vertical (or diapycnal) transport of heat and salt across density surfaces has a strong influence on the water mass distribution, and hence on the long-term circulation. Diffusive parametrizations take the form

$$
q_{T}=\frac{\partial}{\partial z}\left(k_{T} \frac{\partial T}{\partial z}\right)
$$

with a corresponding parametrization for salinity. The overall meridional circulation has been found to depend strongly on the magnitude of the diapycnal diffusivity $k_{T}$, with higher values of $k_{T}$ resulting in a stronger circulation and higher meridional heat transports (Bryan 1987). Values around $10^{-4} \mathrm{~m}^{2} \mathrm{~s}^{-1}$ are necessary to simulate observed transports and water mass distributions. Semi-empirical theories based on the energy budget of turbulent motions suggest that $k_{T}$ should be related to stability measures such as the buoyancy frequency. It is necessary to include vertical convection due to unstable density stratification by enhanced mixing, particularly when modelling water mass formation in high latitudes. It is not well known whether the difference of the molecular diffusivities of heat and salt (which leads to double-diffusion phenomena such as salt fingering) results in different effective values for the respective turbulent coefficients. Special attention has to be given to the oceanic surface layer where wind-generated turbulence provides much higher mixing levels.

The lateral mixing of heat and salt is provided mainly by the mesoscale eddy field (see section 4 below). The large difference in vertical and horizontal turbulent kinetic energy requires a strongly anisotropic diffusivity tensor, with horizontal diffusivities typically around $(1-5) \times 10^{3} \mathrm{~m}^{2} \mathrm{~s}^{-1}$ depending on numerical resolution. Intuitively, an alignment of its principal axes with isopycnal (constant density) surfaces seems more natural than the choice of horizontal/vertical directions as mixing along isopycnals does not immediately affect the available potential energy of the system. Energy arguments alone are not conclusive, however, and the question remains one of the many open issues in subgrid scale parametrization. 
The representation of turbulent momentum fluxes in models which do not resolve mesoscale eddies has followed similar lines, with values for the eddy-induced mixing coefficients which are significantly higher than the corresponding values for heat and salt. The physical basis for these parametrizations is however weak, and analysis of eddy-resolving models has indicated much more complicated patterns of momentum fluxes which cannot be represented by any diffusive approximation. Over large regions, momentum fluxes have turned out to be upgradient, corresponding to negative viscosity coefficients. In models which explicitly resolve mesoscale eddies, biharmonic or higher order formulations are used which constitute a more scale-selective dissipation and allow a better representation of the turbulent energy cascade. Particularly in western boundary currents and in the equatorial regions the circulation is very sensitive to the details of turbulent momentum fluxes whereas in the ocean interior that sensitivity is weaker because of the dominant geostrophic balance.

\subsection{Numerical implementation}

The choice of numerical algorithms is not a merely technical matter. Almost always the resolution has been insufficient to obtain reasonable convergence, i.e. to explicitly resolve all the scales of importance and therefore numerical details can have an important influence on the solutions. Moreover, algorithms may even be chosen to deliberately suppress aspects of the solution which are undesirable for a particular purpose. While such an approach implies that the numerical solution does not truly represent the underlying system, it is of ten a more practical way of excluding nonessential processes than modifying the equations of motion to explicitly exclude them.

The system (2.1)-(2.6) is basically of hyperbolic character although some parts may also require the solution of elliptic subsystems. Most models are based on finite difference methods. Spectral (Galerkin) methods which are rather effective in atmospheric models are generally less suitable due to the irregular shape of ocean basins. Coordinate systems with fixed depth levels are most common. Some models, however, use the variable depth of isopycnic surfaces, a formulation that has advantages when modelling nearly adiabatic processes. Forward time stepping with simple explicit schemes is usually sufficiently accurate as numerical stability criteria normally require time steps much smaller than the time scales of interest. To speed up convergence towards a steady state, asynchronous integration schemes with longer time steps in some equations can be employed (Bryan 1984). Occasionally implicit schemes are used which slow down the phase speeds of fast waves so allowing much larger timesteps. Spatial discretization normally retains basic conservation properties of the continuous system (for energy, heat, salt, and sometimes enstrophy). For long-term integrations when advection of water masses is the crucial mechanism, care must be taken to avoid 'unmixing' of heat and salt by numerical dispersion in coarse-resolution models. This can be achieved by using special advection schemes such as the flux-corrected transport algorithm, but at the expense of economy and/or accuracy (Gerdes et al 1991).

To give an idea of the computational needs for high resolution calculations, some numbers are given for an experiment with a North Atlantic circulation model at approximately $16 \mathrm{~km}$ resolution which is in progress at the time of this review. The model is based on a code written by Cox (1984) which has generously been made available to the ocean modelling community. At each time step, seven variables at nearly $10^{7}$ gridpoints must be calculated and stored. With a time step of 10 minutes, an integration for one model year needs approximately $400 \mathrm{~h} \mathrm{CPU}$ on a Cray-2. To 
bring such a model to complete statistical equilibrium would require $\sim 1000$ years of model integration equivalent to 50 years of Cray-2 time! Presently, integration times of eddy-resolving models are limited to several years, at most a few decades. These integration times allow the circulation to come to a quasi-adjustment with the density field although the latter is still adjusting. Numerical experiments which address processes at longer time scales are still restricted to coarse horizontal resolution (typically $100-400 \mathrm{~km})$. These experiments do not permit explicit resolution of eddies which hence must be parametrized.

\section{Observing the ocean circulation}

\subsection{Instruments and techniques}

No routine observing system for the ocean exists comparable to the global network of meteorological observations, and the lack of sufficient data has been a major obstacle for the verification and development of ocean models. In particular, information on ocean processes occurring deeper than $\sim 500 \mathrm{~m}$ has come exclusively from dedicated experiments which can be made only from specially equipped oceanographic research vessels.

At the heart of classical oceanography is the measurement of temperature and salinity over the depth of the water column which allows inference of profiles of horizontal velocity via geostrophy. Passive tracers of either natural (e.g. nutrients, silicate, $\mathrm{C}^{14}$ ) or anthropogenic (e.g. bomb-tritium or chlorofluorocarbons) origin contain important information on the flow provided that their sources/sinks are sufficiently well known. Hydrographic observations are sparse, however, and the world ocean has not been sampled to an adequate resolution even once. Repeat sections which allow some assessment of temporal variability of the deep ocean exist only in very few places.

Reliable techniques for direct subsurface current observations exist. Deep sea moorings typically contain 5-10 current meters over $5 \mathrm{~km}$ depth and can be left in the water for over a year. Instrumentation is expensive, however, and deployment highly technical, so current meter observations are not abundant. Furthermore, moored instruments provide Eulerian information on the velocity which is usually dominated by strong mesoscale variability so that inference on large-scale circulation patterns can be difficult. Information on the circulation field can also be obtained from freely drifting floats. $\dagger$ At depths around 1-2 km, SOFAR-floats are used which are acoustically tracked in the sound channel, either by listening for sound sources located around the basin or alternatively by emitting sound which is detected at several suitably located listening stations. For deeper velocities a more economical (but less informative) way of tracking floats is being developed where the floats periodically come to the surface and telemeter their position to a satellite.

Another potentially very useful (but also rather expensive) technique for obtaining information on subsurface thermal structure is acoustic tomography which permits the continuous measurement of temperature distributions in certain depth ranges by measuring the travel times of acoustic pulses along various ray paths between moored

\footnotetext{
$\dagger$ Historically, when measurement of deep currents was first attempted in 1959 by following neutrally buoyant floats (Crease 1962, Swallow 1971) there were surprises as the ocean currents turned out to be much swifter and more variable than expected. The floats rapidly dispersed and the experiment as planned was a failure, but it alerted oceanographers to the fact that the ocean in many places is filled with eddies an order of magnitude more energetic than the mean flow.
} 
stations (Knox 1989). It has been applied successfully over scales of several hundred kilometers and can in principle be used for monitoring long-term temperature changes over large distances. Acoustic techniques also appear capable of yielding integral information on the velocity field in the form of the average tangential velocity along a track between two stations which can be combined with other tracks to form a closed area and by Kelvin's circulation theorem to give a value for the average vorticity.

Near the surface, observations are more readily available. Merchant shipping covers a fair proportion of the world's oceans, and it has long been the custom to use the information recorded by such ships to glean information on surface winds, surface temperature and surface currents. $\dagger$ More recently some merchant ships have been instrumented to make $\mathrm{XBT}_{\mathrm{BT}} \neq$ measurements, and these data are fed into operational analyses of the upper ocean, particularly in the tropics. Surface drifters are drogued at a depth of a few tens of metres with a buoy at the surface to telemeter position to a satellite. Depending on the depth of the drogue and the structure of the boundary layer, they measure the wind-induced velocity in the mixed layer or the geostrophic velocity beneath.

Satellite observations are beginning to have an increasing impact on oceanography. While they give information mainly on surface parameters, no other technique has a comparable spatial and temporal coverage. Infrared images which allow the determination of sea surface temperature in cloud-free regions are routinely being used, in conjunction with ship-based observations to produce maps of SsT anomalies on monthly timescales and have also been useful in providing synoptic views of mesoscale and regional structures. But perhaps the most important instrument which ocean modellers are beginning to use systematically is the satellite altimeter. This instrument gives information on the height of the sea surface (which is related to the circulation) by measuring the travel time of a radar pulse between satellite and ocean surface. The instrumental accuracy is very high (typically a few centimetres over a distance of $800 \mathrm{~km}$ ), but there are some environmental factors which require corrections, such as variations of atmospheric water vapour content. One of the main uncertainties comes from the geoid which is not known to a comparable accuracy. If the satellite is in a repeat orbit, then temporal changes in sea surface can be determined without. Another source of error is that the satellite orbit is not very well known. These errors have a large spatial scale and do not seriously degrade the information on scales of tens to hundreds of kilometers so the instrument is particularly suited to mapping the eddy field as manifest at the surface. The first altimeter was launched on the SEASAT satellite in 1978 and transmitted data for three months. More recently the altimeter on the GEOSAT satellite provided data from 1986 to 1989 (see figure 4.1 below). The altimeter has also been used to good effect in the tropical ocean and maps of surface height changes were produced every month before the demise of GEOSAT. In the long term, it is expected that improvements in the measurement of both geoid and the orbit will make the altimeter useful for determining the circulation on longer space/time scales also. Altimeters and other instruments are placed on ERS-1, a European Satellite

$\dagger$ Halley (1686) produced a surprisingly accurate qualitative picture of the wind patterns over the oceans, particularly in the tropics by talking to ship's captains, and Benjamin Franklin similarly documented the existence and location of the Gulf Stream in 1786 .

$\ddagger$ An XBT (expendable bathy-thermograph) is a temperature probe on the end of a very thin wire. As the instrument falls it relays this information along the wire to the ship which does not have to alter its sailing pattern in any way. XBT measurements are less accurate than those from hydrographic casts but are much cheaper and more plentiful and still able to resolve changes in the heat content of the upper ocean to depths of typically $500 \mathrm{~m}$. 
launched in summer 1991, and on TOPEX-POSEIDON, a high accuracy USA/French mission to be launched in 1993.

In section 2, it was noted that the forcing fields for the ocean were inadequately known and that this was a hindrance to verifying/improving ocean models. One instrument which is capable of sensing the wind field near the ocean surface is the satellite microwave scatterometer which measures the back scatter of a radar pulse which is related to the surface waves and hence to the magnitude of the wind. Although not straightforward, it is also possible to obtain wind direction, and it is expected that the scatterometer (which is included in the ERS-1 payload) will, in combination with other information, provide global information on the wind as a main forcing of ocean circulation.

Large-scale experiments within the World Climate Research Program are continuing to enhance our ability to monitor the ocean. The TOGA-program (Tropical Ocean Global Atmosphere) has been very successful in expanding routine wind and nearsurface temperature observations in the tropical oceans, with the focus on interannual climate variations. WOCE (World Ocean Circulation Experiment) is focused on globalscale observations which are necessary for a better understanding of decadal climate change. The ultimate objective is to establish a global ocean observing system, combining satellite observations with in situ ocean measurements, which can be used to monitor climate trends and to help with the development of models for climate simulation and prediction.

\subsection{The use of data with models}

Observations will never be so plentiful that one could hope to understand or deduce the ocean circulation by measurements alone. The information content from measurements must be augmented by ocean dynamics which are embodied most comprehensively in ocean models. So far, the focus of ocean model development has been on prognostic models (as described in section 2) which permit calculation of the oceanic state for given sets of initial boundary conditions. The validation of models with observed data is however more efficient if appropriate techniques for optimally combining data with models are employed.

Such techniques have been developed in many disciplines under a variety of names such as 'inverse modelling', 'optimization', 'data assimilation', or 'variational methods'. The methodologies, while often very similar, differ in purpose, computational technique, and feasibility, but all have two general objectives, namely (i) the accurate estimation of the circulation state, especially of those parameters which are not directly observable, such as transports and fluxes and (ii) the quantitative test of dynamical hypotheses which should ultimately lead to improved formulations of prognostic models. In contrast to the situation with prognostic models, the number of possible approaches to inverse modelling is large. Differences can result not only from different dynamical approximations but also from different types of data and from different inversion algorithms. The problem is a familiar one in meteorology, and the necessities of weather forecasting have led to various practical solutions (see the review by Gilchrist 1988). Inverse modelling in oceanography is much less developed, and applications to ocean circulation problems have been few and these usually restricted to particular data sets.

Applications have tended to fall in one of two categories. On the one hand, sophisticated (and in a statistical sense, optimal) inversion techniques have been used with rather simple models (frequently geostrophic) and comparatively limited data 
sets, mainly from hydrographic observations. This approach has been used especially when trying to deduce the long-term circulation. Specific objectives have included not only the determination of the absolute velocity field and of property budgets, but also of model parameters such as mixing coefficients. From a dynamical perspective, these models are somewhat incomplete as they do not contain a closed set of dynamical equations (which would be required for a complete forward calculation with time evolving flows).

On the other hand, data insertion or assimilation procedures have been used in conjunction with highly sophisticated models. Most frequently, this approach has been used for problems related to mid-latitude mesoscale or tropical interannual variability which in many ways are closest to the meteorological forecast problem. Examples are the assimilation of altimeter data into QG models to follow the evolution of regional current systems such as the Gulf Stream, the assimilation of XBT data into general circulation models of the tropical oceans on a routine basis to monitor the state of the surface layers as part of the TOGA experiment (see also section 5). This approach normally requires only minor modifications of existing models. A general disadvantage is that issues of consistency and errors of the solution are usually not explicitly addressed.

The gap between inverse modelling and data assimilation philosophies will perhaps be bridged by the recent development of adjoint techniques which have become possible due to advances in computing technology. Such methods are not new but have recently attracted considerable interest as they begin to become feasible for applications to large systems. For further details of variational assimilation, see, for example, Courtier and Talagrand (1990) or articles in Anderson and Willebrand (1989). They are particularly appropriate for systems with a large number of variables (e.g., initial or boundary conditions) for which optimum values are sought. The construction of the adjoint to any given system is a relatively straightforward but tedious task (see Thacker and Long (1988), for a brief description of the method). The adjoint equations are integrated backwards in time, with an effort comparable to that of the corresponding forward model. In principle, any kind of observation can be incorporated in those models. As with other related methods, it is necessary to specify a cost function (misfit) which ideally should be based on a priori knowledge of the solution statistics. For the all too common case of underdetermined systems, a priori assumptions on the solution itself are also necessary (they often come disguised as smoothness, or similar, requirements). The adjoint equations are an effective way to compute the gradient of the cost function with respect to all parameters. First experiments with an adjoint of a relatively complete ocean circulation model have been performed, but it is too early to assess the practicability of this method.

The development of techniques which can handle the continuous voluminous fluxes of data which are expected from satellites is especially important. Experience has shown that the establishment of appropriate operational capabilities for monitoring and prediction will be necessary to achieve substantial progress.

\section{Wind-driven circulation and the role of eddies}

The ocean is a driven system, forced by the fluxes of momentum, heat and fresh water. These forcings operate on rather different timescales. It takes an ocean model of the order of 1000 years to reach equilibrium in the deep layers if forced by fluxes of heat 
and fresh water, a timescale that is determined by vertical advection and mixing processes. On the other hand, a model can adjust to wind forcing (with the thermal structure largely imposed) within a few years. For practical reasons then, the wind induced circulation has been studied more than the thermohaline circulation. The early questions related to how the ocean responded to imposed wind forcing, how the gyres were spun up, how eddies developed in different regions of the world's oceans, how topography shaped the currents, their transports, and their transients. Because western boundary currents are highly energetic, they tend to have more interesting dynamics, and considerable attention was devoted to these regions.

The first calculations involved only linear dynamics. The boundary current of the subtropical gyre corresponds to the Gulf Stream, which separated from the coast (often modelled just as a straight coast aligned N-S) at about the right latitude. As nonlinearity was included and the model resolution increased, the boundary current became inertial and extended further north before separating from the coast. As resolution was increased still further, the jet did not retain its laminar inertial character but became unstable and eddies were formed.

During the last decade there has been an intensive effort in eddy-resolving simulations, with models of increasing resolution and complexity. In most cases, QG models with idealized geometrical configurations and wind forcing patterns were used. The thermal stratification was taken as given and not altered, the main purpose of such calculations being to study the influence of eddies on the large-scale circulation, and also the resolution dependence of the simulation. In particular, whether new features appeared as resolution improves, or the Gulf Stream separated correctly, and at what resolution the larger scale flow became insensitive to resolution. A second motivation was to resolve the eddies properly with a view to parameterizing them in coarser resolution climate models.

One of the early uses of QG models was to consider the implications of the constraints of almost-two-dimensional turbulence. $\dagger$ Theory had shown that in two-dimensional turbulence, energy could not be cascaded downscale easily, even though it is at small scales that dissipation will ultimately act. In two-dimensional turbulence, energy should be cascaded primarily upscale while enstrophy (vorticity squared) should be cascaded downscale. Thus, if energy is injected into the fluid at the eddy scales, it will be cascaded to large scale flows which then feel the effect of spherical geometry of the earth. A review of two-dimensional turbulence on the sphere is given in Rhines (1979).

The need for an enstrophy cascade to small scales means that large scale flows must have regions of strong gradients within them. Theories are most conveniently formulated in terms of potential vorticity (PV), which for practical purposes in the ocean can be simplified to $(\xi+f) \partial \rho / \partial z$ where $\xi$ is the relative fluid vorticity and $f=2 \Omega \sin$ (latitude) is the background vorticity of the Earth. For largescale motions this can be simplified further to $f \partial \rho / \partial z$ since $\xi$ is small compared with $f$. From (2.1)-(2.6) it can be deduced that in the absence of forcing and dissipation (which could be both mechanical and thermal), potential vorticity should be conserved following the fluid motion. Because PV is a scalar quantity, with some analogy to a passive tracer, its behaviour is somewhast easier to understand than that of other dynamic quantities. A passive tracer that is advected along closed streamlines around a largely

$\dagger$ Although the atmosphere and ocean are three dimensional, their vertical extent is rather limited compared to the scales of horizontal excursions and so, to first order, the flow can sometimes be considered as two dimensional. 
horizontal gyre would become uniform in the centre of the gyre, provided that the mixing is sufficiently small. Extrapolating back to potential vorticity then suggests that it too should be uniform in the centre of the wind-driven gyres, at those depths below which there is no direct atmospheric forcing.

Several interesting results were found from QG calculations with a two-gyre (subtropical and subpolar) configuration (see Holland et al 1984). In the surface layer there are strong gradients of potential vorticity reflecting the wind forcing with above average potential vorticity in the northern gyre, below average in the southern gyre. In layers beneath the surface, potential voritcity was indeed found to be uniform over a large region of the basin spanning both gyres, with the size of the regions of homogenized PV decreasing with depth. Although there is no mean forcing in subsurface layers, a mean circulation is obtained which is driven by a systematic downward momentum transfer by the eddies. This transfer is most intense in the regions close to the western boundary to north and south of the Gulf Stream. It is remarkable that the recirculation gyres are not visible in the depth of isopycnic surfaces, indicating that they are not geostrophic and would not be detected from geostrophic calculations with observed $T-S$ fields.

The numerical calculations further showed that the level of eddy kinetic energy, its geographical extent and connection to the penetration scale of the jet are all strongly related and sensitive to a number of model parameters. The zonal penetration of the jet is a balance between the inertial character which tends to cause the jet to extend far to the east and a counter tendency due to the instability of the jet which tends to limit its eastward penetration. For example, increasing explicit friction tends to make the jet extend further east, since the growth of eddy activity and the self braking effect which this produces on the jet is reduced. In this respect the eastward penetration is similar to the northward penetration of the western boundary current referred to earlier.

The main deficiency in QG calculations is their inability to properly model thermal forcing. In recent years, eddy-resolving calculations with PE-models have become feasible which allow a fuller representation of diabatic processes. A pioneering study was performed by Cox (1985), with an idealized configuration resembling the North Atlantic. More recently, eddy-resolving calculations with realistic forcing and geometry have become possible, and Semtner and Chervin (1988) have performed a global calculation with half-degree resolution. A one-third-degree model of the North Atlantic has formed the basis of the WOCE community modelling effort (Bryan and Holland 1989). These calculations allow a direct comparison with observations. Figure 4.1 demonstrates that the horizontal pattern of eddy energy in that model agrees quite well with the altimetric observations. The amplitudes are however too small, particularly in higher latitudes, indicating that eddies are still incompletely simulated at this resolution. This is not surprising as the Rossby deformation radius, the preferred scale of instability, decreases to $10 \mathrm{~km}$ at high latitudes. An experiment by Böning and Budich (1992) has indeed confirmed that the eddy energy increases substantially when the resolution is increased to one-sixth degree especially in high latitudes and in the near-surface layers where it is approximately doubled.

High resolution models, even when forced with strictly periodic annual fluxes exhibit interesting low frequency variability with time scales of several years. There is some indication that a geostrophic-turbulence cascade is operative producing large scale patterns, modulated by the variation in Coriolis parameter, which give rise to Rossby wave activity, but integration times have so far been too short to clearly identify the cause of this variability. 

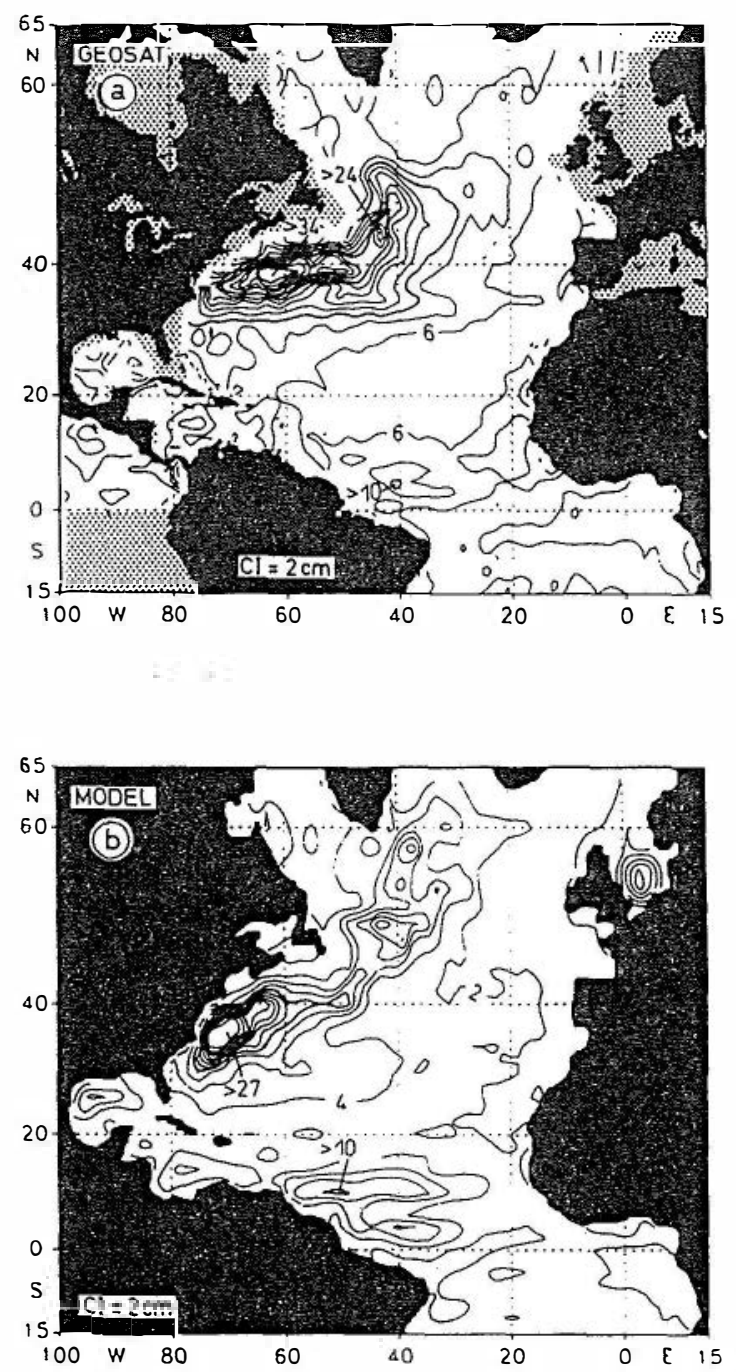

Figure 4.1. Root-mean-square elevation of the sea surface in the North Atlantic, $(a)$ as measured from the GEOSAT altimeter during 1987-89 and $(b)$ as calculated from the COCE community model. Units are in $\mathrm{cm}$. The horizontal patterns of the model variability agree fairly well with the observations, and show highest energies in the region of the Gulf Stream and its extension. The model amplitudes, however, are in general significantly smaller than the observed values, indicating that model resolution is still insufficient. (From Stammer and Böning 1992.)

From the viewpoint of climate modelling, a question of high practical importance is whether or not eddies must be included in climate calculations. Figure (from C Böning, private communication) shows potential vorticity on a density surface from two model integrations, one at one-third degree and a control run at one degree resolution. In the eddy resolving model, the explicit coefficients of mixing are reduced but the effective mixing, accomplished by the eddies, is clearly enhanced. In the high-resolution case there is a fairly large region of weaker PV gradients but it is not nearly as uniform as in the (somewhat unrealistic) QG simulations. A process competing 


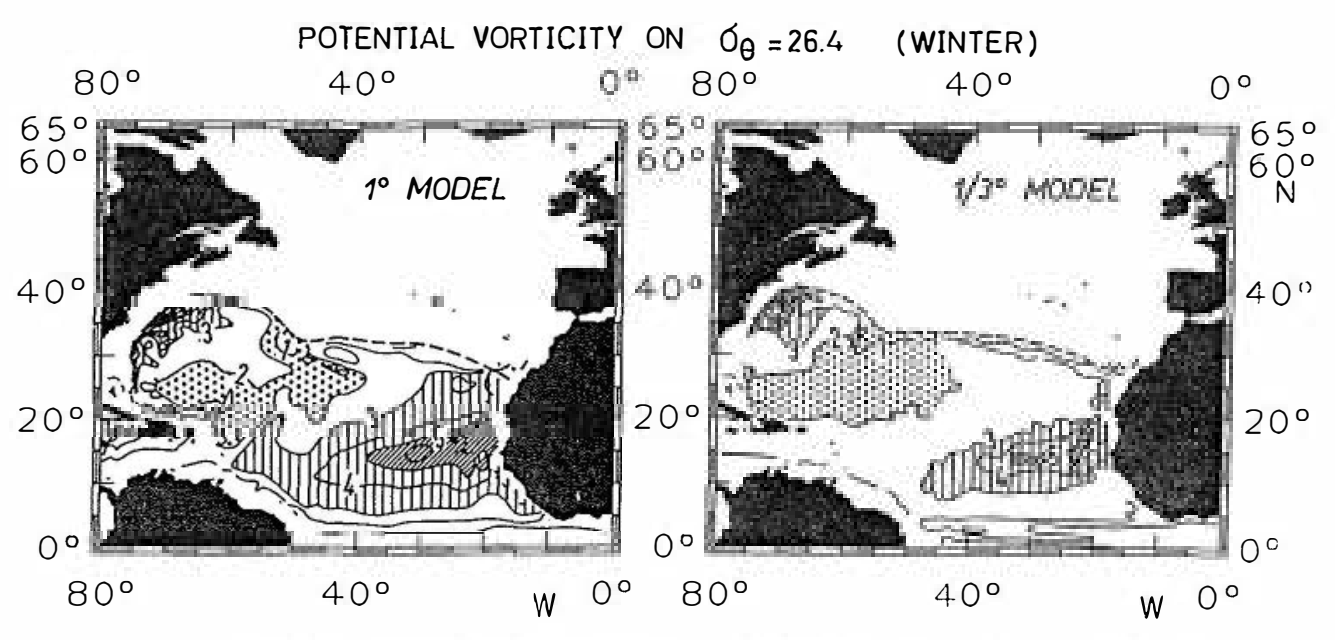

Figure 4.2. Potential vorticity on the $\sigma=26.4$ density surface from two models of the North Atlantic. Eddies are parametrized in the one-degree calculation but explicitly resolved in the one-third degree model. The effective mixing in the eddy-resolving model is clearly enhanced and results in a fairly large region of weaker PV gradients to the coarse-resolution case. The ventilation region and unventilated ('shadow') zones in the Eastern basin are less pronounced in the high-resolution model. Strong PV gradients occur in the NorthWestern region where the density surface outcrops and is exposed to atmospheric cooling. (Courtesy C Böning.)

with PV homogenization is the thermal forcing which is most noticeable in the northwestern region where the density surface outcrops and is exposed to atmospheric cooling. To the east of the homogenized region in figure 4.2 is a region of higher PV gradients which is not directly reached by water previously exposed to the surf ace and is bypassed by the flow field. This unventilated region (shadow zone) which had been expected on theoretical grounds (Luyten et al. 1983) is less pronounced in the highresolution calculation. It is hence clear that the eddies are important for mixing of tracers entering the main thermocline, including in particular a temperature signal caused by atmospheric warming. Accurate modelling of the oceanic response to greenhouse warming therefore requires appropriate representation of the eddies, either explicitly or parametrically.

The meridional heat transport, a variable associated with ocean climate on longer time scales, seems however to be less influenced by eddies. The direct contribution of eddies to heat transport is of order $0.1-0.2 \mathrm{PW}$, roughly $10 \%$ of the maximum total transport. The eddy transport is equatorward in the subtropical gyre, and poleward in higher latitudes. There is also an indirect contribution through eddy-induced changes of mean flow and temperature distribution. Cox (1985), with a model where isotherms coincide with constant density surfaces, found that both contributions nearly compensate so that the total transport is hardly affected by eddies. Preliminary results from the community model seem to confirm no significant large-scale changes in heat transport due to the eddies. In higher latitudes where the total ocean heat transport is smaller, the relative contribution of the eddies can however be more important, and there are substantial regional differences associated with a northward shift of the subpolar front. Results concerning heat transport in these models must still be interpreted with some caution as the integration times have not yet been sufficient to obtain thermal equilibrium. 
A special region in the ocean where the presence of eddies dominates the dynamics is the Antarctic Circumpolar Current (ACC). The overall eddy energy is larger here than in other parts of the world ocean. The dynamical regime is quite different from that in other basins due to the lack of meridional boundaries and the associated western boundary currents. Simulations with idealized QG models (Treguier and McWilliams 1990, Wolf et al 1991) have indicated that the ACC transport which is observed to be approximately $120 \mathrm{~Sv}$ is controlled by the interaction of eddy-induced vertical momentum transfer with large-scale topographic features. This process cannot easily be represented in coarse-resolution models as it requires friction parameters of unrealistically high magnitude. A first experiment with a one-quarter degree PE-model of the ACC has been performed at several UK institutions. First results indicate that the lateral distribution of eddy energy is modelled well whereas the total transport is considerably larger than observed values. The reasons for this discrepancy are not clear but it is not impossible that observed estimates are low in this highly structured current.

\section{Short-term climate variability}

\subsection{A description of ENSO}

Understanding of the meteorological aspects of ENSO started with the work of Gilbert Walker, the Director of the Indian Meteorological Service, who, motivated by the great droughts of 1871 and 1899, sought to predict the strength of the Indian Monsoon (see Walker 1928, and earlier references cited therein). He failed to find a predictor for the monsoon but he did discover a large-scale interannual variation in the surface pressure difference between Western and Eastern Pacific which he called the Southern Oscillation (so), to distinguish it from another (less important) Northern Oscillation. Figure 5.1 shows a modern correlation of pressure at Darwin with that at other stations around the world indicating the large-scale nature of the oscillation. It extends throughout the tropical regions as well as into higher latitudes. The so is now known to be strongly related to SST in the Pacific as shown in figure 5.2 from Rasmusson (1984). Shading corresponds to ENSO years when the equatorial temperatures are anomalously warm, which happens every three to five years.

Interannual variations in currents and sea surface temperatures in the Eastern Equatorial Pacific Ocean had been known since at least the end of the last century but it was not till Bjerknes (1966) that a plausible connection was made between variations in SST in the Equatorial Pacific and the Southern Oscillation. The East-West (E-W) temperature gradient along the Equator varies interannually and Bjerknes argued that this was related to winds along the Equator. When Eastern SST is cold, there is a strong thermal gradient along the Equator. The warm water in the West is associated with rising motion in the atmosphere and the cold water in the East with descending motion. Since the Coriolis parameter in the Equatorial zone is small, the atmospheric flow will be largely down the pressure gradient (rather than across it as at mid-latitudes) giving rise to an E-W circulation shown schematically in figure 5.3. Weakening the E-W thermal gradient leads to a weakening of the winds and to a reduced atmospheric pressure difference between the East and West Equatorial Pacific.

This notion is clearly incomplete since it gives no indication of a cycle between warm and cold events but it still lies at the heart of the ENSO mechanism. A missing ingredient is how the ocean adjusts to changing winds. The adjustment is accomplished 
Correlations of Annual Mean Sea Level Pressures with Darwin

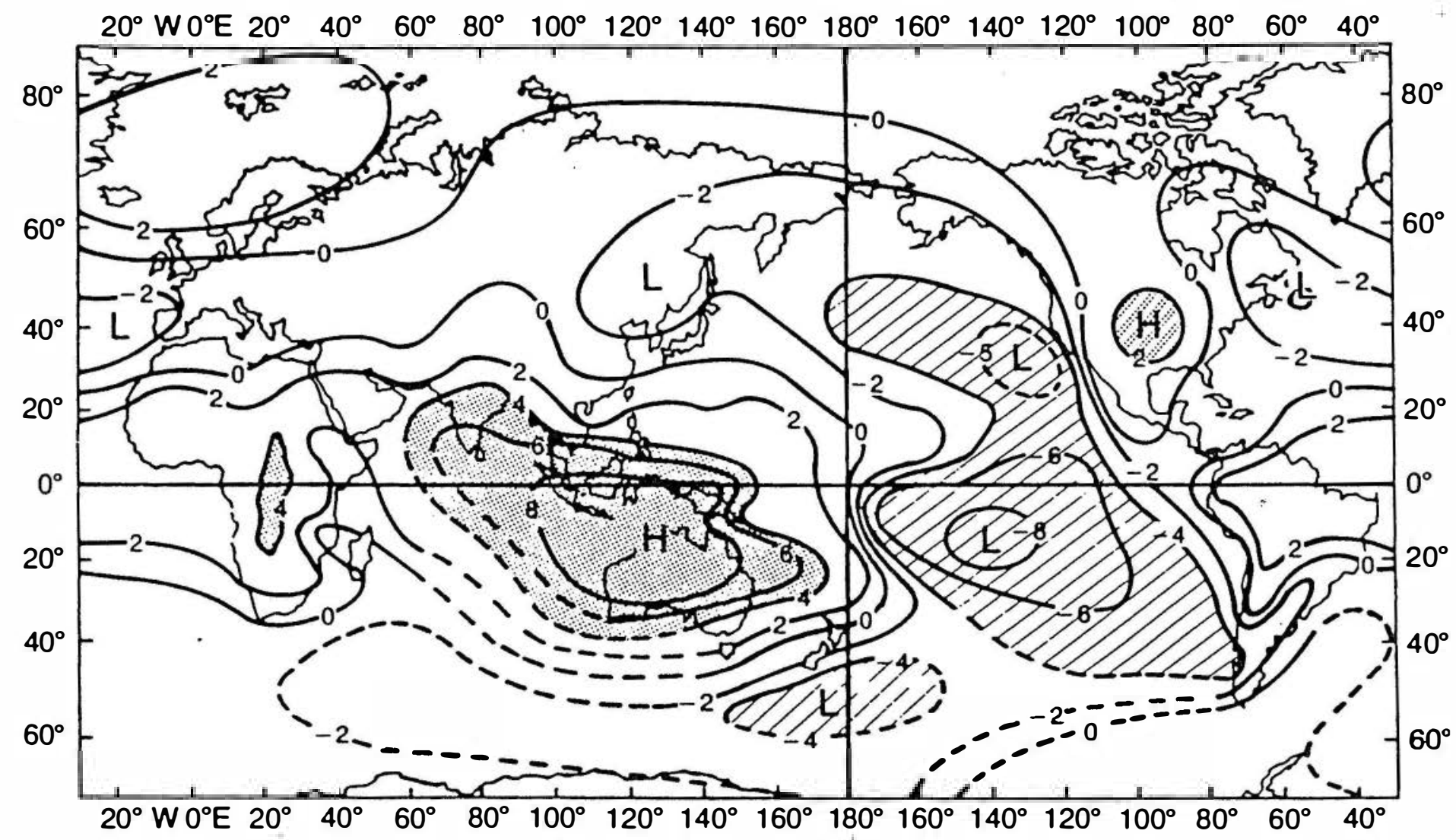

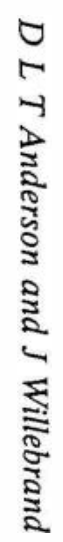

Figure 5.1. Plot of the correlations of surface pressure at Darwin, Australia, with the pressure around the world showing the huge spatial scale of ENSO. When pressure is high in the West Pacific, it is low in the East Pacific. Although there are connections to mid-latitudes they are less robust. Units are scaled such that a value of 10 indicates perfect correlation. From Trenberth and Shea (1987). 


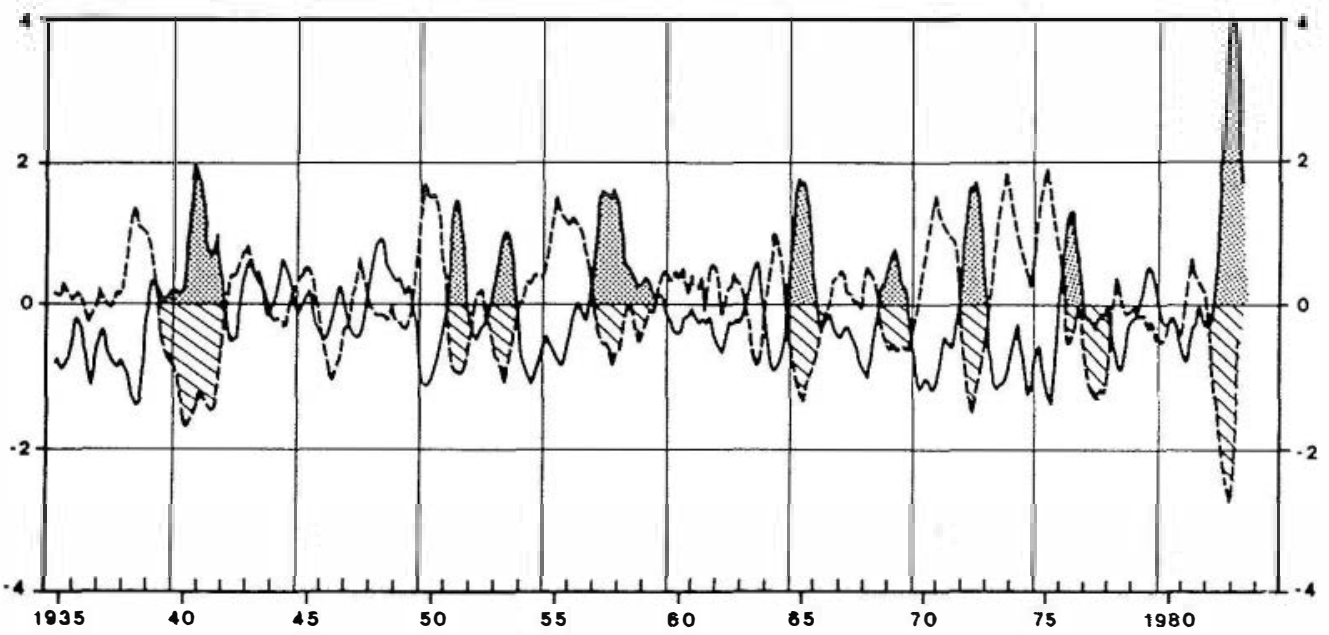

Figure 5.2. Plot of SST (solid line) and the Southern Oscillation index (dashed line) over the last 50 years showing the strong connection between what is hapenning in the ocean and changes in the atmosphere. Shading indicates ENSO events which occur typically every few years without being periodic. From Rasmusson (1984).

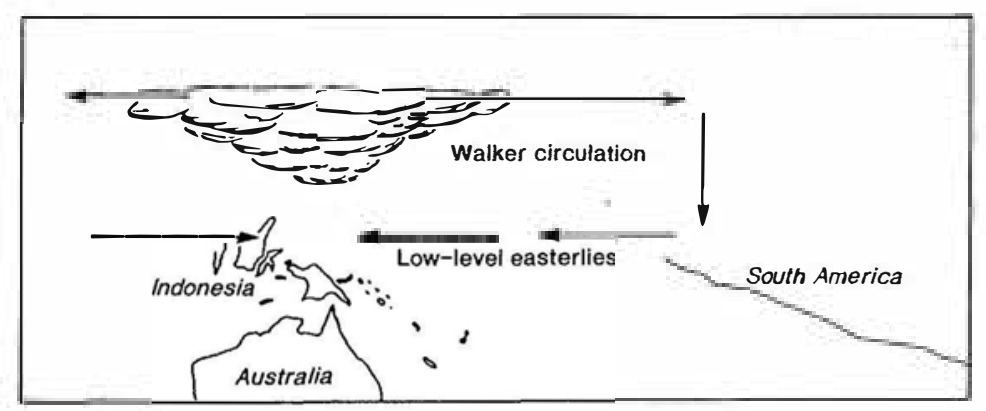

Figure 5.3. A schematic to show the vertical circulation along the equator. No air parcel will actually follow the streamlines shown since there are also meridional circulations which are not shown. The main idea of the cartoon is to indicate rising motion over the maritime continent (Indonesia), fed at low levels both by the Easterly winds of the Pacific ocean and the Westerly winds of the Indian ocean. This region of rising motion is associated with heavy precipitation. Further to the east there is descending motion over the colder waters of the easter Pacific. These waters are in turn cold because the surface winds are Easterly, pulling the thermocline to the surface in the East.

by certain types of waves, notably Rossby waves and Kelvin waves, which have wavelengths of several thousand kilometres and can propagate long distances in the ocean without crippling attenuation. Kelvin waves propagate along boundaries or Eastward along the Equator, while Rossby waves, at the wavelengths of interest, carry their energy to the West. A number of numerical studies in the early eighties were devoted to illustrating how these waves were excited, propagated, and could be focused or superimposed to give wave beams (see McCreary 1985 for a review). More recently, it has been confirmed observationally, from satellite measurements of sea surface displacements and in situ measurements in the ocean, that Kelvin waves can travel across the width of the Equatorial Pacific $(15000 \mathrm{~km})$. It has been more difficult to observe long-wavelength Rossby waves which carry information to the West because 
they have speeds comparable with mean currents and hence are likely to be affected by the mean flow. The propagation of Rossby and Kelvin waves which are important in climate change is controlled by the density stratification in the subsurface ocean which has a sharp thermocline at depths around $100 \mathrm{~m}$. There is a whole variety of wave speeds associated with these waves depending on their vertical stratification and, in the case of Rossby waves, how far they extend from the Equator. Typical speeds for the free Kelvin waves are from 3 to $1 \mathrm{~ms}^{-1}$ and for the Rossby waves from 1 to $0.1 \mathrm{~m} \mathrm{~s}^{-1}$. The fastest Kelvin wave can cross the basin in $\sim 2$ months (fast compared with ENSO timescales) while Rossby waves take an order of magnitude longer.

A plausible mechanism for obtaining the 3-5 year time scale of ENSO could then involve wave processes, and this idea was embodied in early theories of ENSO (McCreary 1983). The atmospheric components of the wind field used to drive the early models were specified: the ocean-atmosphere interaction resided solely in the mechanism by which the wind fields were activated. This was based on the ideas that when the thermocline in the East Pacific was shallow, then the Walker circulation was strong. When the thermocline was deep, off-Equatorial Westerly winds were strong and excited Rossby waves which then travelled to the Western boundary and reflected as equatorial Kelvin waves.

Although the early models oscillated at ENSO time scales and involved Rossby waves probably in a qualitatively correct way, the form of interaction between atmosphere and ocean was oversimplified. Schopf and Suarez (1988) expanded the wave scenario and combined it with the idea of an unstable interaction region in which the wind and SST strongly influence each other (illustrated schematically in figure 5.4). A background wind is assumed to exist and to have set up an East-West thermal gradient and thermocline slope along the Equator. If this is perturbed by, say, an eastward wind anomaly in the central East Pacific, the thermocline will be depressed to the east, raising SST which will further strengthen the anomaly, giving rise to positive feedback much as in the Bjerknes scenario and embodied in the $a T$ term in the pedagogical equation (5.1) below.

However, these same winds will also excite a Rossby wave which will propagate westward, reflect from the western boundary as an Equatorial Kelvin wave, and return to the interaction region. These are waves of elevation of the thermocline so the reflected Kelvin wave will act to reduce and even reverse the growing disturbance, giving rise

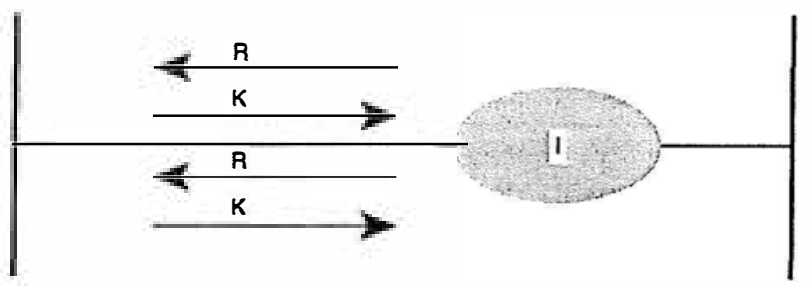

Figure 5.4. A schematic showing the idea of how the timescale of a few years, characteristic of ENSO can arise. The 'long' timescale is associated with the Westwards propagation of Rossby waves excited in an interaction region in mid-ocean. The Rossby waves reflect off the Westerly boundary and return Eastward along the Equator. When they enter the region in which the atmosphere and ocean interact strongly they reverse the interaction and move the system to the opposite phase of the cycle, giving rise to Rossby waves of opposite sign. This schematic implies interaction occurs only in the one key region, which is unlikely to be the case in reality. Its purpose is only to indicate the role of waves in a simplified system, although the model of Cane et al (1986) does appear to conform to it to some degree. 
eventually to a negative SST anomaly and an easterly wind stress. This signals the start of the cold phase, the generation of a Rossby wave of depression which in turn will give rise to the next warm phase and so on. Suarez and Schopf proposed the following conceptual model equation to represent these processes

$$
\frac{\partial T}{\partial t}=a T-c T(t-\delta)-b T^{3}
$$

where $a$ represents the positive feedback term, $c$ the amplitude of the returning signal, and $\delta$ the time delay introduced by Rossby and Kelvin waves, with the nonlinear term acting to limit the amplitude of the perturbation. This simple equation can give rise to a fascinating range of different behaviours, depending on the values of the parameters $a, b, c, \delta$. It can illustrate stable multiple equilibria, and oscillatory solutions, as well as stable equilibria coexistent with oscillatory solutions. It also shows that the period of the oscillations need not be close to $2 \delta$ (where $\delta$ is the time it takes a Rossby-Kelvin wave to make its transit through the Western Pacific) if the parameters correspond to a point near the boundary between stable and oscillatory solutions. Although this equation is only heuristically derived, much of the behaviour suggested by it may be observed in more complicated models. (The reader may want to consult McCreary and Anderson (1991), who have tried to classify the behaviour of many complicated models in terms of the ideas of this and other similar simple equations.)

At the heart of any theory of an ENSO cycle, is the notion that one needs a deep warm pool in the West Pacific producing an increase in the heat content of the western equatorial region before an ENSO can start, i.e. a preconditioning phase. In the above scenario, this build up of heat is accomplished by Rossby waves which depress the thermocline in the West Pacific. An alternative scenario has been envisaged by Anderson and McCreary (1985) in which the huge atmospheric heat source over Indonesia leads to the restoration of easterly winds along the Equator which induce a deep thermocline in the West Pacific. The relative importance of this mechanism vis- $\grave{a}$-vis the RossbyKelvin wave propagation mechanism is unclear and it is quite possible that their relative importance varies from one ENSO event to another or that both processes could be important at different times in the same ENSO event.

\subsection{Modelling ENSO}

Several numerical models have been developed with which to study the ENSO problem. The models range from those which include as many physically important processes as possible called General Circulation Models through those which simplify one component, usually the atmosphere, to those which simplify both. If the simplification is not too drastic, these latter models are called intermediate models to indicate that their complexity is less than that of a GCM. They may still be rather involved, requiring considerable ingenuity in the parametrization of processes handled in a brute force method by a GCM, but they will be considerably less computationally demanding.

Models may be run either in a mode which seeks to simulate the whole interaction, i.e. the mean state of the atmosphere and upper layer $(\sim 300 \mathrm{~m})$ of the ocean as well as the variability, or in a mode where only the variability is simulated. Ideally, one would prefer the former, but the latter has the advantage of reducing the possibility of climate drift which is a consequence of errors in the oceanic and atmospheric components of the coupled model. In particular, if the atmospheric model is forced with observed ssTs and the momentum heat and fresh water fluxes from this model 
are then used to drive an ocean model, one would not reproduce in the ocean model the SSTs used to force the atmospheric model in the first place. In practice it means that if a coupled model were started from a state close to the present, it would drift into a state whose climate is different. Climate drift need not be large to cause serious problems in coupled models. For example, Gordon (1989) has shown that the error in simulated SST in the West Pacific in his model is only of the order of $1^{\circ} \mathrm{C}$, which seems rather a small error and one might conclude that the model really does rather well. In some sense this is true but $1{ }^{\circ} \mathrm{C}$ is actually a serious error as this region is one in which SST gradients are small and the value is already high making it a region to which the atmosphere is particularly sensitive. In fact, the location of strong convection and hence rainfall are substantially in error as a result of this $1^{\circ} \mathrm{C}$ sst error. In a subsequent run, in which the parametrization of mixing of heat in the ocean was altered to allow heat to penetrate a little more deeply, the over-warm water in the West Pacific did not develop and in this respect the later simulation was an improvement. No variability comparable to ENSO was generated, however, for reasons which are not yet clear.

Clouds are thought to play a nontrivial role in ENSO: as SST rises, convection increases producing more clouds which reduce the solar energy reaching the surface. The net heat flux at the surface is reduced, so tending to limit the ssT rise. $t$ Ultimately, any credible model for climate change should predict cloud amount. As an interim step some models use a specified cloud amount, and one such model (Philander et al $1991)$ is able to simulate ENSO variability.

The model was integrated for 28 years and exhibits interesting interannual variability similar to that of ENSO. Figure 5.5 shows the SST patterns during periods of warm and cold events, which apart from the presence of water in excess of $30^{\circ} \mathrm{C}$ are rather similar to what is observed, and occur with approximately the correct time scales. SST anomalies tend to develop in the central East Pacific much as observed $\ddagger$ Although SST anomalies occur in situ, there is still evidence for propagation in other fields, eastwards along the Equator and Westwards at $6^{\circ} \mathrm{N}$ which is quantitatively consistent with the scenario given in figure 5.4 but is not a proof of it. In particular the speed at which information propagates along the Equator is much slower than that of a Kelvin wave. It is tempting to speculate why this model oscillates in a rather realistic way while the model of Gordon does not. Experience from intermediate models, however, indicates that coupled models are very sensitive to small parameter changes which can lead to large changes in the character of oscillations or to the model not oscillating at all.

The most successful perturbation model is that developed by Cane et al. (1986). The atmospheric model is assumed to be in equilibrium with the slowly evolving ocean and therefore totally adjusted to the ocean SST. $\S$ The ocean model has two active layers overlying a deep inert ocean. The thermodynamics and dynamics are independent:

† Other components of the heat flux may change too. The evaporative flux is reduced as the winds become lighter. The net outgoing long wave flux decreases as the backward long wave flux is increased but any change in this is likely to be smaller than in the short wave. The net effect in the heat flux is thus likely to be in such a direction as to limit SST growth.

$\doteqdot$ The ENSO of $1982 / 3$, the largest on record was an exception in this respect in that warm water first appeared in the West Pacific and moved Eastward.

$\S$ This assumption is quite reasonable since atmospheric adjustment times are short compared to oceanic adjustment times. Most coupled models of intermediate complexity have such an assumption built in as this leads to an order of magnitude reduction in computing time. Some models allow time dependence in the moisture equation at a modest additional cost. 
(a) SST

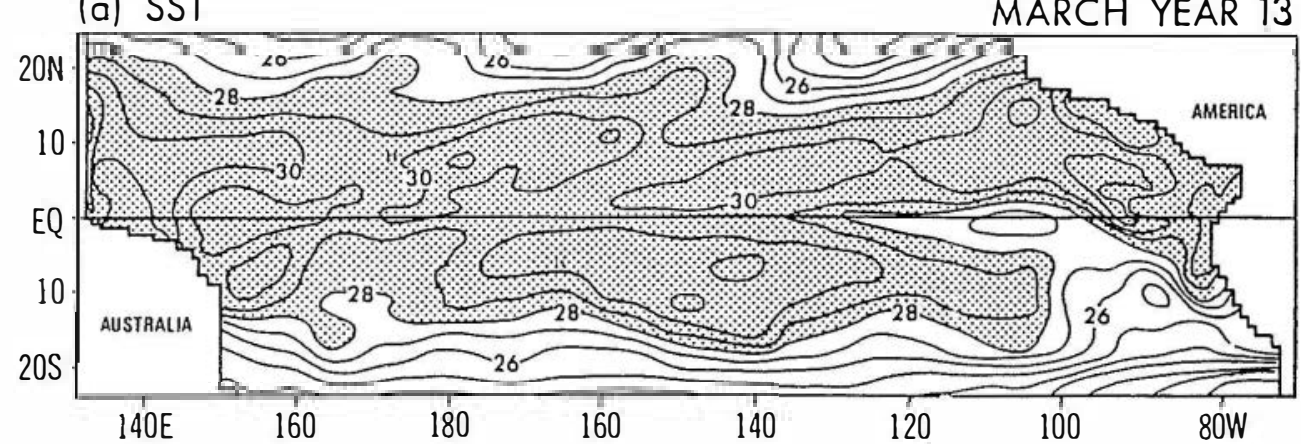

(b) SST

JUNE YEAR 15

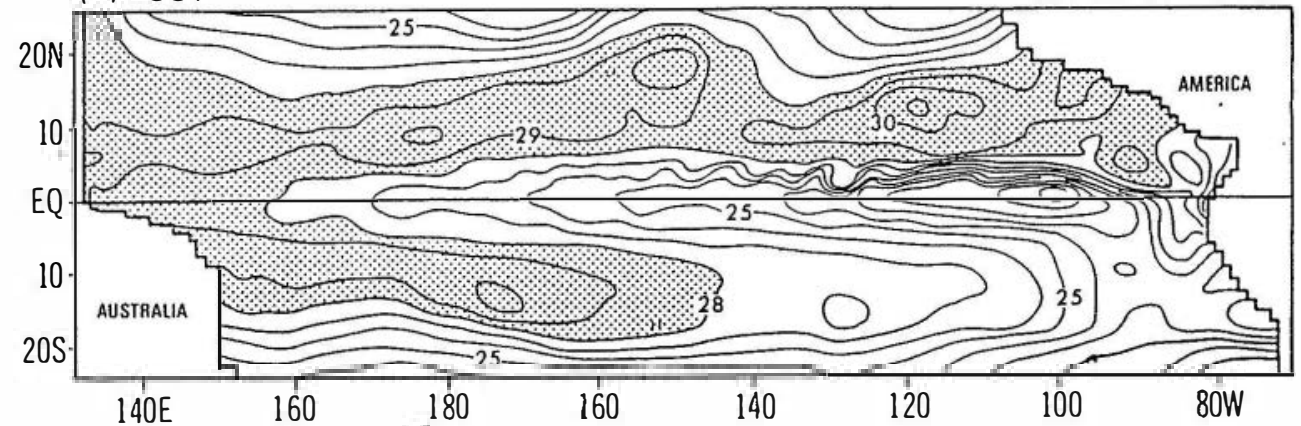

Figure 5.5. SST from a solution to a coupled GCM at the peak of a warm event (upper panel) and at a peak of a cold event (lower panel). Shaded regions indicate temperatures greater than $28^{\circ} \mathrm{C}$. Note the pronounced cold equatorial tongue during the cold event and the appearance of tropical instability waves just North of the Equator in the Eastern basin. Water with a temperature of $30^{\circ} \mathrm{C}$ floods the Eastern ocean during the warm event. (From Philander et al 1991.)

currents are induced as a result of wind forcing only and while these currents alter the SST, changes in SST do not influence the dynamics. An approximation in the meridional momentum equation eliminates fast gravity waves (except the Kelvin wave), allowing longer time steps and hence the possibility of long integration periods extending over hundreds of years. Parameter space can then be explored rather fully, more so than for almost any other model. Spontaneous ENSO events develop with a dominant time scale of 3-4 years, but there are periods of many years without ENSO events and other times when they occur rather regularly, not too unlike the observed record of figure 5.2. The results are somewhat sensitive to model parameters in that an increase in the coupling leads to larger amplitude oscillations and longer periods and vice versa. As in the coupled GCM of Philander et al (1991) and in the study by Latif and Flïgel (1991) and Latif and Graham (1991), ENSO events are preceded by a deep thermocline or build up of heat content in the West Pacific.

\subsection{Predicting ENSO}

The wave-oscillator idea embodied in figure 5.4 suggests that ENSO events should be exactly repeating and therefore infinitely predictable. This idea is clearly not correct. As figure 5.2 shows there is a considerable spread in the time between events. Figure 
5.4 also implies that there is one canonical ENSO evolution whereas each event is observed to be different. The difference could arise for a number of reasons. Variability in the atmosphere whose cause is not related to the tropical Pacific ocean (loosely called stochastic forcing, although it could also include more coherent large-scale tropical disturbances such as the intraseasonal oscillation with time scales of months) might be important. Other possibilities are inherent nonlinearities in the oscillator, reminiscent of chaotic behaviour as expressed by Lorenz (1976), or interaction between ENSO and other processes causing climate variability such as the quasi-biennial oscillation, snow cover variations over the Eurasian land mass or variability in the ocean outside the Pacific region.

Nonetheless, one might conjecture that if one could somehow determine where the 'system' was on the oscillator, it might be possible to predict subsequent events. And even if the oscillator idea of figure 5.4 is not correct there may yet be some predictive skill for other reasons. Cane et al. (1986) were the first to attempt climate forecasts using their perturbation model. The results were encouraging and forecasts are now made on a regular (three month) basis and published in the Climate Analysis Bulletint. Figure 5.6 shows the two year forecasts made over the 1976 and 1982 ENSO. In each case, six forecasts were made starting from different consecutive months. As panel (a) shows these forecasts can sometimes differ showing a strong sensitivity to initial conditions, so it has been found to be advantageous to average forecasts over the initial conditions to give a consensus forecast.

There are only a relatively few ENSO events for which there is an adequate amount of data to verify any model forecast. Testing a model is therefore rather difficult and problematical since it is verified on only a few realizations and frequently (as in figure 5.6) verified against only one parameter namely SsT in the central Eastern Equatorial Pacific. Nonetheless, figure 5.6 shows that for the 1982-1983 event the skill was considerable. This is true for the 1976 event as well as for the non-event period 1977-1979. (It is just as important that the model does not predict an event when there is not one as that it does predict an event when there is.) The model skill appears to be greatest in predicting major events and almost zero in predicting the month to month variability in SsT.

An interesting feature which has emerged is that the skill is seasonally variable. Forecasts for the Northern Hemisphere winter period tend to be more skillful which is thought to be a consequence of the stability of the mean state changing seasonally. In spring/summer, the coupling between atmosphere and ocean is strongest and the forecast suffers most from errors in initial conditions at this time.

This raises the question as to how the initial conditions are determined. The tropical ocean, driven by prescribed forcing is a largely deterministic system, by which is meant that if the forcing were known perfectly and the ocean model were perfect, then one could determine the ocean state simply by running an ocean model with prescribed forcing. $\neq$ To a large degree, ENSO is thought to involve adiabatic processes which can be embodied in the wind field. In the case of the model of Cane et al (1986) the ocean component is preconditioned by forcing it with just the past history of the wind

† This is a bulletin published monthly by the Climate Analyses Centre of NOAA which contains an up to date analysis of many tropical fields, both atmospheric and oceanic.

$\ddagger$ The tropical ocean is not totally deterministic because there are spontaneous instabilities to the north of the equator giving rise to eddies there (called Legeckis waves). These eddies are quite small scale and therefore not likely to be of importance in influencing climate, except possibly in an ensemble sense by contributing to meridional heat transport. 

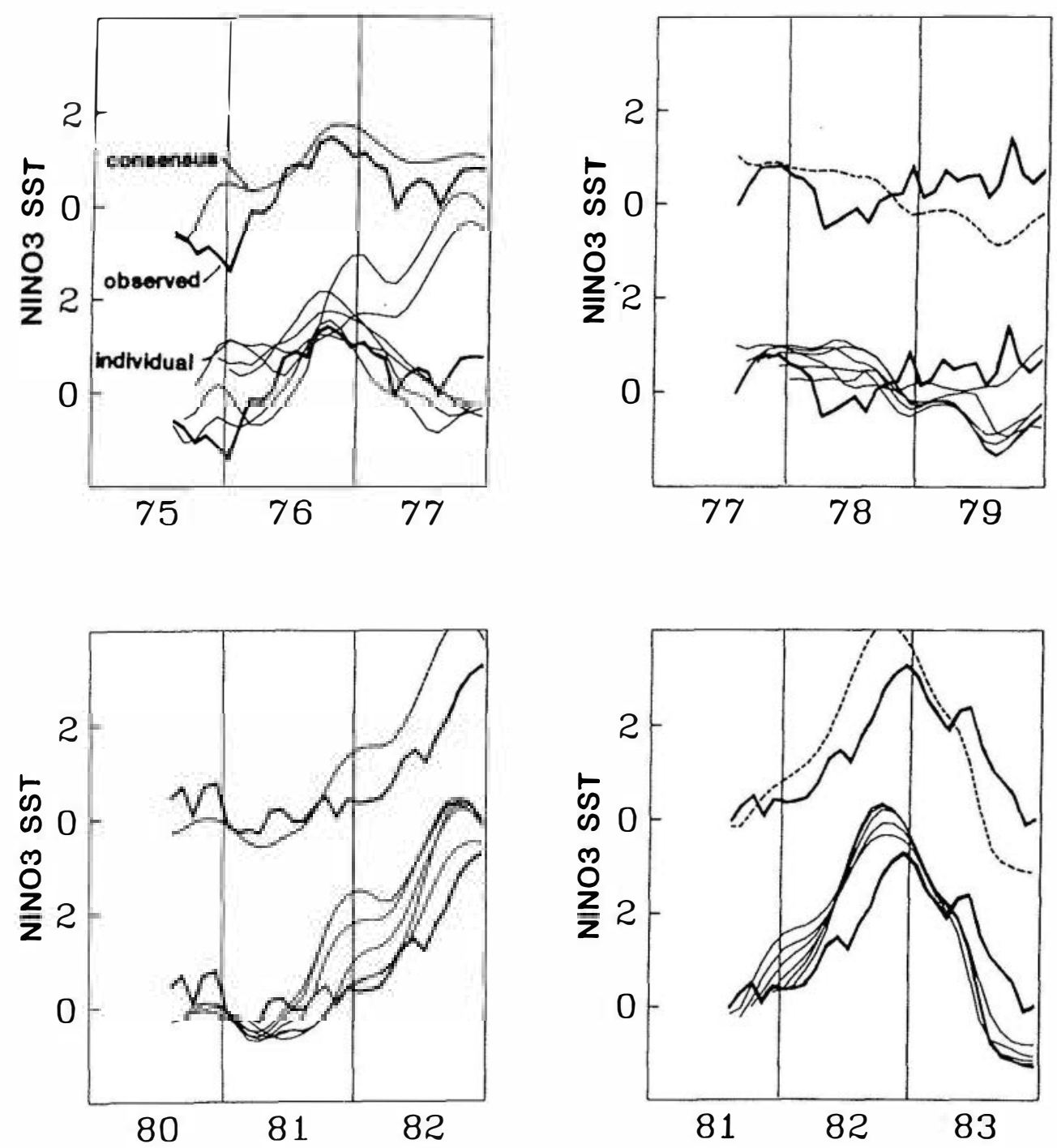

Figure 5.6. SST anomalies $\left({ }^{\circ} \mathrm{C}\right)$ for selected periods over the central-east equatorial Pacific. Light curves are from hindcasts initiated in the six successive months from August to the following January. The dashed curve gives the average of the six, i.e. the concensus forecast while the heavy curve gives the observed values. Note the level of agreement between the solid and dashed curves for the 1976 and 1982 ENSO periods.

field: thermal information is not used so the model thermal state may differ from the observed. Errors in the thermal state may often decay over the first few months, in others they may grow. This depends on the nature of the errors and on stability of the coupled system. If it is close to a stability boundary, then it may be particularly sensitive to initial errors. The growth of errors has been considered in some detail by Goswani and Shukla (1990) over a twenty-four year period who show that the model does sometimes hindcast an ENSO event when none occurs. They also find a systematic error in the model with the largest errors occurring in forecasts of the decaying months of warm events. 
An ocean model has also been used to analyse the patterns of ENSO variability and in particular to look for predictors (Latif and Flügel 1991). Ideally one would simply analyse the data to determine optimum predictors, but unfortunately records especially of subsurface thermal data do not go back far enough to permit this. Nonetheless, one can generate pseudo-data, for example, model temperatures in the upper $300 \mathrm{~m}$ obtained by forcing the ocean model with observed wind stresses over the period 1961-1985, and analyse those. Seeking the principal oscillation pattern for equatorial thermal data reveals a mode which starts with warm water in the West Pacific some nine months before the height of an ENSO event followed by warm water in the East Pacific during ENSO and cold water in the West Pacific nine months after that. The interesting feature is that there is very little signature in the temperature field at the surface in the West: it is located mainly at $200 \mathrm{~m}$ below the surface. This warm water at depth will however lead to a rise in sea level and some precursor in sea level has been noted by Wyrtki (1985). The difficulty in using the upper ocean thermal field of the Western Pacific as a predictor is that it seems to be a necessary but not sufficient pre-condition for ENSO. A rise in sea level does not always lead to an ENSO, nor is the amplitude related to the strength of the subsequent ENSO. As in the previously cited study, the forecast skill is a strong function of season, as shown in figure 5.7.

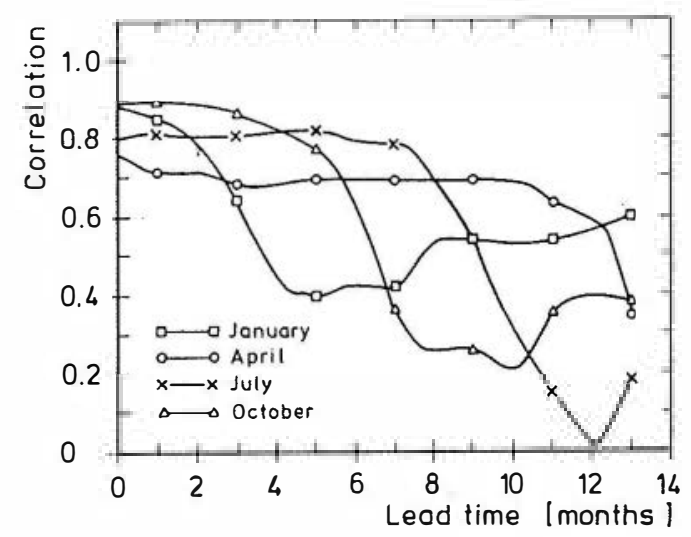

Figure 5.7. Correlation of equatorial temperature with SST in the central equatorial region as a function of lead time. The correlations show a strong seasonal dependence. Temperatures in April are much better predictors than those in january. From Latif and Graham (1991).

The models are far short of the potential capabilities of coupled GCMs, so we might reasonably expect an improvement in forecast skill as the GCMs are improved to the extent that they can be used for prediction. While model improvement is a necessary step, it alone is not sufficient. The atmosphere has limited predictability as the largest time scale of coherent atmospheric motion in the tropics is the intraseasonal oscillation with a lifetime of a month or two. The information on which an ENSO climate forecast is based lies in the slowly evolving boundary conditions, primarily ocean SST, but to some extent land conditions also. Therefore, a necessary ingredient for a good forecast is a good ocean initial state. This cannot be obtained solely from atmospheric forcing fields because these are inaccurate, particularly with respect to the heat flux: an ocean analysis is also required, using all relevant ocean observations. How to use data poses interesting technical and procedural issues. This was discussed briefly in section 3.2. 
Leetmaa and Ji (1989), Thacker and Long (1988), Derber and Rosati (1989) and Sheinbaum and Anderson (1990) have considered techniques for assimilation of data within the tropical region relevant to TOGA.

\section{Thermohaline circulation and long-term variability}

The water mass distribution in the world ocean and the associated thermohaline circulation are forced by fluxes of heat and fresh water at the sea surface. The wind forcing, however, cannot be decoupled from thermohaline forcing as both forcing mechanisms influence the circulation field and also the temperature and salinity fields.

For a long time, the main emphasis in global models has been on the steady-state circulation. To achieve equilibrium by forward integration requires integration times of more than one thousand model years. The first experiment with a fully thermodynamic global model (Cox 1975) permitted an integration over only two years. Faster computers and the use of special asynchronous integration techniques soon made equilibrium calculations possible. Presently, about half a dozen different global models are in use, most of them similar in structure to that of Bryan and Lewis (1979), with horizontal resolution typically $100-400 \mathrm{~km}$ and $10-15$ levels in the vertical.

When forced with observed surface temperatures and salinities according to (2.9), the models have been moderately successful in reproducing the large-scale aspects of observed circulation patterns and water mass distributions. Due to their coarse resolution, intense boundary currents are necessarily broader and weaker than observed, and only the total transports are meaningful. Heat transport in most models has been somewhat lower than that inferred from observations. A problem has been that the main thermocline is often too deep and too diffuse. In this respect the model by Maier-Reimer and Hasselmann (1987) shows a remarkably better performance than other simulations. Although not a directly observable variable, the total meridional transport integrated zonally across ocean basins is a very useful diagnostic of large-scale models as it gives an indication of the pathways of water masses. As documented by Toggweiler et al. (1989), models differ considerably in the amount and location of deep-water formation and in the strength of the deep-ocean circulation. Those differences imply different transport properties which can significantly influence the interpretation of results in models of the carbon cycle.

Tracer distributions provide a very sensitive test as to how well models reproduce the pathways of water masses, as first demonstrated by Sarmiento (1983) who found that the modelled distribution of bomb-produced tritium was more sensitive to mixing in high latitudes than were temperature and salinity fields. Models for radiocarbon in the world ocean have succeeded in reproducing qualitatively the observed distribution although quantitative differences remain (see Toggweiler et al 1989).

A particularly interesting aspect of the global circulation which is reasonably well reproduced in simulations is the different behaviour of the Atlantic and Pacific Oceans. The northern North Atlantic is warmer (about $3^{\circ} \mathrm{C}$ ) and more salty, and is the source region of North Atlantic Deep Water which can be traced through all deep ocean basins. The low surface salinities in the North Pacific prevent sinking and the deep water there is the oldest in the world ocean $\left(\mathrm{C}^{14}\right.$ ages which measure the time since a particle was last in contact with the surface layer are 1500-2000 years). Above the main thermocline there is a series of return flows forming what has been called the oceanic 'conveyor belt' (Gordon 1986). In the South Atlantic, this northward flow of 
warm water causes an equator-ward (i.e. upgradient) heat transport of approximately $0.5 \times 10^{15} \mathrm{~W}$ which is transported into the North Atlantic where it is ultimately released into the atmosphere.

In recent years, the question of possible long-term changes in the large-scale circulation has become increasingly interesting. The paleoclimatological record indicates that the conveyor-belt pattern has occasionally been disrupted. The best known example is the Younger Dryas Event, approximately 10000 years ago, when a sudden cooling occurred on a time scale of 100 years or less. There is evidence that during this period the deep water formation in the North Atlantic nearly stopped. The thermohaline circulation and poleward heat transport were much reduced, causing a significant climate change in Europe and North America. It has been speculated (Broecker et al. 1985) that this event could be interpreted as a transition between different equilibrium states of the ocean circulation.

The clue to understanding the possibility of multiple equilibrium states comes from the different way temperature and salinity interact with the atmosphere, as expressed by the boundary condition (2.8). Temperature and salinity have opposing effects on the density. At high latitudes, the buoyancy loss due to cooling normally dominates the buoyancy gain from lower salinities due to excess precipitation, and the density increase causes a sinking to deeper levels and a poleward motion near the surface. However, changes in high-latitude surface salinity tend to be amplified due to a positive feedback mechanism: a decrease in salinity diminishes the equator-pole density contrast and this results in a decrease in the meridional circulation, thereby reducing the poleward transport of relatively salty water from lower latitudes which in turn, further decreases the polar salinity. On the other hand temperatures are not affected in this adjustment since there is strong thermal feed back. The most simple conceptual models describing this mechanism predict two stable equilibrium states in each hemisphere, one having strong poleward heat transport, with relatively warm and salty water and sinking at high latitudes, while the other one is colder and fresher, has no sinking and a small equator-ward heat transport (Stommel 1961, Rooth 1982).

A judicious choice of the thermohaline boundary conditions is essential for obtaining results from ocean models in an uncoupled mode. Bryan (1986) first demonstrated the existence of multiple equilibrium states in a $3 \mathrm{D}$ general circulation model, using an idealized 2-hemisphere configuration forced by net fresh water fluxes and specified surface temperatures, on the assumption that the timescale of atmospheric interactions is very short. His experiments confirmed the positive feedback suggested by simple models as the principal reason for multiple equilibrium states, and in addition pointed out the important role of convection in high latitudes which greatly increases the sensitivity of the circulation. Manabe and Stouffer (1988) have found two stable states in a global coupled ocean-atmosphere model using a flux correction technique to obtain more realistic precipitation patterns. Besides the presently observed conveyor belt circulation, a second solution was found which has a much colder (up to $5{ }^{\circ} \mathrm{C}$ ) and fresher (three parts per thousand reduction in salinity concentration) northern North Atlantic and much weaker heat transport, with less significant changes in the Pacific circulation. Corresponding differences occur in the atmospheric climate, in particular in the Northern Hemisphere.

It is not yet known whether or not other equilibrium states for the global circulation exist. A series of experiments using a global ocean model with idealized geometry has identified at least four stable equilibrium states (Marotzke and Willebrand 1991). Two of these states represent conveyor-belt circulations, one corresponding to the present 
circulation state and one where the respective roles of Atlantic and Pacific oceans are reversed. Two further states exist where the thermohaline circulation in both oceans is aligned, i.e. sinking occurs either in both northern or both southern basins. Figure 6.1 shows the global ocean heat transport for all four states. The solutions labelled I, II and III all have strong poleward heat transport in the northern hemisphere and weak transport in the southern, while IV behaves differently with very weak transport in the northern hemisphere. While the surface temperature in these simulations was fixed, it is clear that in coupled experiments high-latitude sinking will coincide with higher than normal temperatures.

The existence of multiple equilibrium states immediately poses the question of their stability, i.e. what kind and magnitude of perturbation is necessary to turn the system into a different state. So far we have only some rather preliminary results from uncoupled ocean models. Maier-Reimer and Mikolajewicz (1989) investigated the stability of the North Atlantic circulation to local freshwater input, in an attempt to model the Younger Dryas Event as a consequence of increased melt water inflow through rivers. They found a high sensitivity to both the strength and the location of the freshwater input and an abrupt breakdown of circulation and heat transport within a few decades (see figure 6.2). This fast response occurs because the fresher surface water suppresses the convection in high latitudes very effectively (the polar halocline catastrophy first reported by Bryan 1986). Qualitatively, this breakdown corresponds to a transition from equilibrium I to IV in figure 6.1. It is remarkable that fresh water fluxes of $0.02 \mathrm{~Sv}\left(1 \mathrm{~Sv}=10^{6} \mathrm{~m}^{3} \mathrm{~s}^{-1}\right)$ were sufficient to trigger the event. Marotzke and Willebrand (1991) found that moderate changes in the large-scale precipitation patterns were also sufficient to cause a transition between equilibrium states.

Changes in high-latitude surface salinity are not the only possible way to perturb thermohaline circulation patterns. Bryan and Spelman (1985) calculated the response of a coupled ocean-atmosphere model to an instantaneous quadrupling of the atmospheric $\mathrm{CO}_{2}$ concentration. While this is not a very realistic scenario, the results were nevertheless intriguing. Only twenty-five years after the increased $\mathrm{CO}_{2}$ concentration

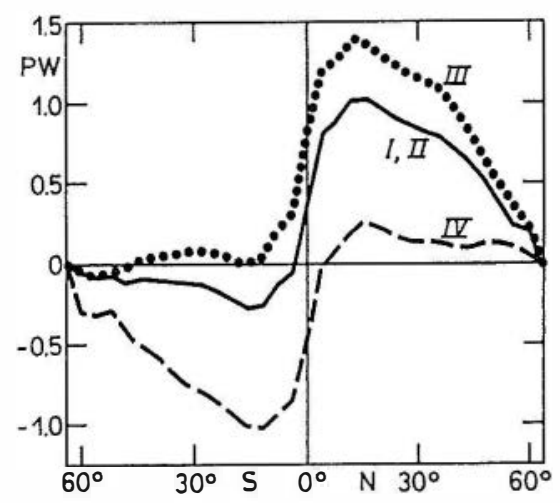

Figure 6.1. Northward transport of heat for different equilibrium states in a global ocean circulation model. As a consequence of the idealized symmetric geometry, both conveyor belt solutions have identical total heat transport (curve I + II). A further equilibrium state (curve III) has sinking in both Northern basins and enhanced Northwood transport. The curve labelled IV belongs to a circulation pattern where deep water is formed only in the Southern hemisphere, and heat transport is directed Southward. It corresponds qualitatively to the second equilibrium state found by Manabe and Stouffer (1988). 


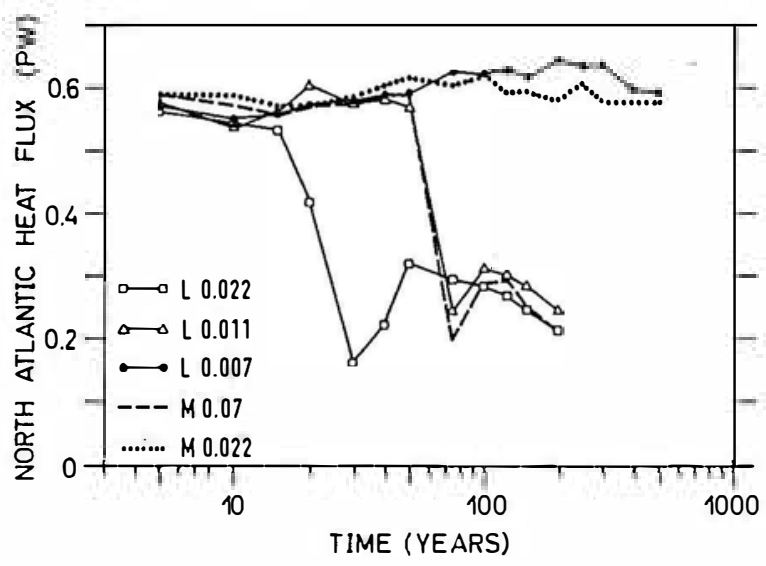

Figure 6.2. Response of an ocean model to injections of meltwater. Shown is the heat flux out of the North Atlantic north of $33^{\circ} \mathrm{N}$. The numbers indicate the strength of the meltwater inflow in Sverdrup, a measure of volume flow equivalent to 1 million cubic metres per second. $\mathrm{L}$ and $\mathrm{M}$ denote release at the $\mathrm{St}$ Lawrence river and the Mississippi, respectively. Whether and when the circulation collapses depends on both the strength and location of the fresh water injection. After Maier-Reimer and Mikolajewicz (1989).

was switched on, the thermohaline cell had almost completely collapsed. A recent study by Mikolajewicz et al. (1990) calculated the ocean response to a gradual $\mathrm{CO}_{2}$ induced atmospheric warming. The increased heating was strong enough to decrease the transport of the vertical cell, and the reduction of convective mixing caused a cooling of sea surface temperatures in regions of water mass formation in the North Atlantic.

The apparent possibility that the thermohaline circulation can collapse within a few decades has an obvious relevance for internal variability on decadal and longer time scales. Positive feedback mechanisms such as the one described above can trigger instabilities of the circulation resulting in oscillatory phenomena. Welander (1986) has given an overview on other possible instability mechanisms. Some indications of internal instability mechanisms leading to limit cycles and chaotic behaviour have recently been found in experiments with idealized GCMs. Marotzke (1990) found that one of the equilibrium states of a global model exhibited regular self sustaining-oscillations with a period of around 25 years. Both the period and amplitude of the oscillation were highly sensitive to details of the simulation, and some equilibrium states did not show any oscillatory behaviour. Similar results are reported by Weaver and Sarachik (1991), who also found aperiodic fluctuations involving strongly increased deep ocean ventilation occurring at scales between 200 and 1000 years.

Another source of variability is stochastic forcing by high-frequency variability in either the atmosphere or the ocean, related to atmospheric weather or mesoscale eddies in the ocean. As shown by Frankignoul and Hasselmann (1976), this type of forcing can generate oceanic variability on much longer time scales than that of the forcing, leading to a generally red spectrum of oceanic variables. Only recently the exploration of long-term variability with ocean circulation models has become feasible. Mikolajewicz and Maier-Reimer (1990) have simulated fluctuations in precipitation and evaporation patterns by adding a random white-noise component to the freshwater fluxes driving their global ocean model. From an integration over more than 3000 model years they found strong variations on time scales around 300 years which is 


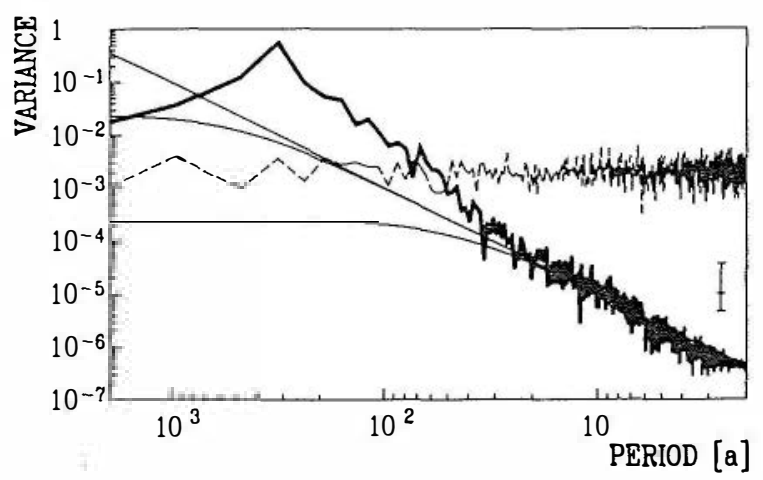

Figure 6.3. Plot of the variance spectrum of the mass transport through the Drake passage (heavy full line) from an ocean model forced with random white-noise surface freshwater flux (spectrum shown as dashed line). As a result of ocean dynamics, the model has maximum variability at periods around 300 years which is highly correlated with North Atlantic heat transport. For comparison the ocean response expected from a simple linear model with time constants of 50 years, 500 years and infinite, respectively, are also shown (thin lines). From Mikolajewicz and Maier-Reimer (1990).

approximately the ventilation time for the deep Atlantic Ocean. Figure 6.3 shows the variability spectrum of the mass transport through the Drake Passage which is highly correlated with the heat transport in the North Atlantic. It exhibits a maximum at a period of 320 years. The main process associated with this maximum is a recurring change in the ventilation of the deep Atlantic Ocean, with salinity anomalies propagating from north to south. The variations in deep water formation reach nearly $50 \%$, associated with heat flux changes of $0.2 \mathrm{PW}$. Those amplitudes depend on the (somewhat arbitrary) choice of the strength and scales of random forcing which corresponded to $16 \mathrm{~mm}$ per month RMS precipitation, with lateral scales of a few thousand $\mathrm{km}$. This choice gives a sizable response but is not strong enough to cause the flip to a different steady state. Analogous experiments by Weaver et al (1991) also resulted in strong variability on decadal time scales.

Numerical studies with stand-alone ocean models have illuminated some aspects of the dynamics of the thermohaline circulation on decadal and longer time scales. With respect to the variability of the ocean-atmosphere system these findings are not conclusive, however, and systematic variability studies with coupled ocean-atmosphere models are needed to properly model these phenomena. We are only at the beginning of understanding the dynamics of longer term variability.

\section{Concluding remarks}

The last few years have seen significant progress in various aspects of modelling the ocean circulation. A decade ago, there were no models capable of resolving the meso-scale (at 30-100 km) ocean eddy field simultaneously with the thermal structure. Today, this has become feasible for basins of the size of the Atlantic, and even for the global ocean. Integration times are still limited to a few decades, a timescale long enough to address questions relating to the dynamics of eddies but too short to consider how the eddies influence the mean stratification. The role of eddies in mixing water masses and passive tracers is important, whereas their effect on the large-scale transport properties seems less pronounced although this issue is still under some debate. 
A decade ago, there was no comprehensivve theory of short-term climate variability such as ENSO. Largely as a result of the deployment of a tropical ocean observing system, of a clearer understanding of how waves in the ocean interact with the atmosphere, and of improved ability to model atmosphere-ocean interactions, this situation has changed to the extent that climate forecasts, albeit rudimentary, are now possible for the Pacific region. In the next few years many coupled general circulation models will evolve which are capable of simulating ENSO variability. We are at a stage where weather forecasting was perhaps in the 1950s and can expect that useful and reasonably detailed forecasts for certain regions of the tropics will become possible. In middle latitudes, the situation is in some respects easier, but in others much more difficult. The atmosphere in mid-latitudes is considerably more turbulent than in the tropics and much of this turbulence on monthly and seasonal time scales is chaotic and non-predictable. There is however some suggestion that large-scale anomalies in mid-latitude sst cause a predictable response in the atmosphere. There is also evidence that climate variations in the tropics may impose a predictable response in middle latitudes in certain circumstances. Changes in SsT in mid-latitudes are mainly locally induced as the waves which are so important for ENSO either do not exist here or are too slow to become significant. While it is not yet known how large the coherent response is compared to the unpredictable part, one can anticipate an expansion in coupled model activity directed to the objective of global-scale climate predictions on seasonal time scales.

Ten years ago, the question of whether or not the thermohaline circulation might have multiple stable equilibrium states did not receive much attention (although a theory for the fundamental mechanism already existed). Since then, results from a number of quite different model studies indicate that this is indeed very likely and can have serious consequences for long-term climate predictions. Most of these studies have been incomplete as they have not accurately represented atmospheric feedbacks. Yet the possibility that the ocean may flip from one state to another within a few decades is intriguing, and the issue will become more important as attempts are made to understand climate variability on decadal and longer time scales. Long-term integrations with coupled models are only beginning as the atmospheric component is computationally much more expensive than the ocean. We can expect, however, that such computations will gradually become possible, and that a close symbiosis between oceanic and atmospheric modelling will be established.

This review has attempted to give some flavour of the excitement new possibilities in modelling and observations have generated in the field of ocean modelling. Significant uncertainties in the formulation of models remain, particularly with regard to the parametrization of small-scale processes and to the surface fluxes. Progress can come only from a systematic validation with observations, and most likely will require the development of appropriate inverse modelling algorithms. The next decade will see the ocean recognized as an integral part of the climate system. Large scale comprehensive observational programmes are in place or being formulated to observe the ocean in a way not possible a decade ago.

\section{Acknowledgments}

We thank C Böning, R Hide and W Schattke for a critical review of the manuscript. 
This work was partly supported by NERC under grant number GR3/7366A and by the Deutsche Forschungsgemeinschaft (SFB 133).

\section{References}

Anderson D L T and McCreary J P 1985 The role of the Indian Ocean in a coupled ocean-atmosphere model J. Atmos. Sci. 42 2439-42

Anderson D L T and Willebrand J (eds) 1989 Oceanic Circulation Models. Combining Data and Dynamics (Dordrecht: Kluwer)

Bacastow R and Maier-Reimer E 1990 Ocean circulation model of the carbon cycle Climate Dynamics 4 95-125

Bjerknes J 1966 A possible response of the atmospheric Hadley circulation to equatorial anomalies of ocean temperature Tellus 18 820-9

Böning C W and Budich R 1992 Eddy dynamics in primitive equation models: sensitivity to horizontal resolution and friction J. Phys. Oceanogr. in press

Broecker W S, Peteet D M and Rind D 1985 Does the ocean-atmosphere system have more of operation? Nature 315 21-6

Bryan F 1986 High-latitude salinity and interhemispheric thermohaline circulation Nature 323 301-4

1987 Parameter sensitivity of primitive-equation ocean general circulation models J. Phys. Oceanogr. 17 970-85

Bryan F and Holland W R 1989 A high-resolution simulation of the wind- and thermohaline-driven circulation in the North Atlantic Ocean. In Parametrization of small-scale processes (ed P Müller and D Henderson) Proc. 'Aha Huliko' a Hawaiian Winter Workshop, University of Hawaii at Manoa 17-20 January 1989, 99-115

Bryan K 1969 A numerical method for the study of the circulation of the World Ocean J.. Comput. Phys. 4 $347-76$

- 1984 Accelerating the convergence of ocean-climate models J. Phys. Oceanogr. 14 666-73

Bryan K and Lewis L J 1979 A water mass model of the world ocean J. Geophys. Res. 84 2503-17

Bryan K and Spelman M J 1985 The ocean's response to a $\mathrm{CO}_{2}$-induced warming J. Geophys. Res. 90 No. C6 $11679-88$

Cane M A, Zebiak S E and Dolan S C 1986 Experimental forecasts of El Niño Nature 321 827-32

Courtier P and Talagrand O 1990 Variational assimilation of meteorological observations with the direct and adjoint shallow water equations Tellus 42A 531-49

Cox M D 1984 A primitive equation, 3-dimensional model of the ocean GFDL Ocean Group Technical Report No. 1 GFDL/Princeton University

_ 1985 An eddy resolving numerical model of the ventilated thermocline J. Phys. Oceanogr. 15 1312-24

Crease J 1962 Velocity measurements in the deep water of the western North Atlantic J. Geophys. Res. 67 $3173-6$

Derber J and Rosati A 1989 A global oceanic data assimilation scheme J. Phys. Oceanogr. 19 1333-47

Enfield D B 1989 El Niño, past and present Rev. Geophys. 27 159-87

Frankignoul C and Hasselmann K 1976 Stochastic climate models, part II. Application to sea surface temperature anomalies and thermocline variability Tellus 29 289-305

Gerdes R, Koeberle C and Willebrand J 1991 The influence of numerical advection schemes on the results of ocean general circulation models Climate Dynamics 5 211-26

Gilchrist A 1988 Numerical weather prediction and climate simulation Rep. Prog. Phys. 51 1205-26

Gill A E 1982 Atmosphere Ocean Dynamics (New York: Academic) 662 pp

Gordon A L 1986 Interocean exchange of thermocline water J. Geophys. Res. 91 5037-46

Gordon C 1989 Tropical ocean-atmosphere interactions in a coupled model Phil Trans. R. Soc. A 329 207-23

Halley E 1786 A historical account of the trade winds and monsoons observable in the seas between and near the Tropicks, with an attempt to assign the phisical cause of the said winds Phil Trans. R. Soc. 16 $153-68$

Hansen J and Lebedeff S 1988 Global surface air temperatures: update through 1987 Geophys. Res. Lett. $15323-6$

Holland W R 1978 The role of mesoscale eddies in the general circulation of ocean-numerical experiments using a wind-driven quasi-geostrophic model J. Phys. Oceanogr. 8 363-92

Holland W R, Keffer T and Rhines P B 1984 Dynamics of the oceanic general circulation: the potential vorticity field Nature 308 698-705 
Holloway G 1989 Subgridscale representation. Modelling the Ocean General Circulation and Geochemical Tracer Transport ed D L T Anderson and J Willebrand (Dordrecht: Reidel) 513-94

Knox R A 1989 Ocean acoustic tomography: a primer Modelling the Ocean General Circulation and Geochemical Tracer Transport ed D L T Anderson and J Willebrand (Dordrecht: Reidel) 141-88

Latif M and Flügel M 1991 An investigation of short range climate predictability in the tropical Pacific J. Geophys. Res. 96 C2 2661-73

Latif $M$ and Graham N 1991 How much predictive skill is contained in the thermal structure of an OGCM? J. Phys. Ocean submitted

Leetmaa A and Ji M 1989 Operational hindcasting of the tropical Pacific Dyn. Atm. Oceans. 13 465-90

Lorenz E N 1976 Nondeterministic theories of climate change Quarternary Res. 6 495-506

Luyten J R, Pedlosky J and Stommel H 1983 The ventilated thermocline J. Phys. Oceanogr. 13 292-309

Maier-Reimer E and Hasselmann K 1987 Transport and storage of $\mathrm{CO}_{2}$ in the ocean-an inorganic ocean-circulation carbon cycle model Climate Dynamics 2 63-90

Maier-Reimer E and Mikola jewicz U 1989. Experiments with an OGCM on the cause of the Younger Dryas Oceanography ed A Ayala-Castanares, W Wooster and A Yanez-Arancibia Mexico City UNAM Press $87-100$

Manabe S and Stouffer R J 1988 Two stable equilibria of a coupled ocean-atmosphere model J. climate 1 841-66

Marotzke J 1990 Instabilities and multiple equilibria of the thermohaline circulation Ber. Inst. Meeresk., Kiel 194126 pp

Marotzke J and Willebrand J 1991 Multiple equilibria of the global thermohaline circulation J. Phys. Oceanogr. 21 1372-85

McCreary J P 1983 A model of tropical ocean-atmosphere interaction Mon. Weather Rev. 111 370-89

1985 Modeling equatorial ocean circulation Ann Rev. Fluid Mech. 17 359-409

McCreary J P and Anderson D L T 1991 An overview of coupled ocean-atmosphere model of El Niño and the Southern Oscillation J. Geophys. Res. 96 3125-50

McWilliams J C, Norton N J, Gent P R and Haidvogel D B 1990 A linear balance model of wind-driven, midlatitude ocean circulation J. Phys. Oceanogr. 20 1349-78

Mikolajewicz U and Maier-Reimer E 1990 Internal secular variability in an ocean general circulation model Climate Dynamics 4 145-56

Mikolajewicz U, Santer B D and Maier-Reimer E 1990 Ocean response to greenhouse warming Nature 345 589-93

O'Brien J J (ed) 1986 Advanced Physical Oceanographic Numerical Modeling (Dordrecht: Reidel)

Parker D E, Folland C K and Ward M N 1988 Sea-surface temperature anomaly patterns and prediction of seasonal rainfall in the Sahel region of Africa Recent Climate Change (New York: Belhaven) $166-78$

Philander S G H 1990 El Nirio and the Southern Oscillation (New York: Academic)

Philander S G H, Pacanowski R C, Lau N C and Nath M J 1991 A simulation of the Southern Oscillation. Part II: Results from high-resolution coupled general circulation models of the ocean and atmosphere J. Climate (submitted for publication)

Preisendorfer R 1988 Principal component analysis in meteorology and oceanography Developments in Atmospheric Science Vol 17 (Amsterdam: Elsevier)

Rasmusson E 1984 El Niño: The ocean-atmosphere connection Oceanus 27 5-13

Rhines P B 1979 Geostrophic turbulence Ann. Rev. Fluid Mech. 11 401-11

Rooth C G H 1982 Hydrology and ocean circulation Progr. Oceanogr. 11 131-49

Sarmiento J L 1983 A simulation of bomb tritium entry into the North Atlantic J. Phys. Oceanogr. 13 1924-39 Schopf P S and Suarez M J 1988 Vacillations in a coupled ocean-atmosphere model J. Atmos. Sci. 45 549-66

Semtner A J and Chervin R M 1988 A simulation of the global ocean circulation with resolved eddies J. Geophys. Res. 93 15502-22

Sheinbaum J and Anderson D L T 1990 Variational assimilation of XBT data. Part II: Sensitivity studies and use of smoothing constraints J. Phys. Oceanogr. 20 689-704

Stammer D and Böning C 1992 Mesoscale variability in the Atlantic Ocean from GEOSTAT altimetry and WOCE high resolution numerical modelling $J$. Phys. Oceanogr. in press

Stommel H 1961 Thermohaline convection with two stable regimes of flow Tellus 13 224-30

Swallow M 1971 The Aries current measurements in the western North Atlantic Phil. Trans. R. Soc. 270 451-60

Tans P P, Fung I Y and Takahashi T 1990 Observational constraints on the global atmospheric $\mathrm{CO}_{2}$ budget Science 247 1431-8

Thacker W C and Long R B 1988 Fitting dynamics to data J. Geophys. Res 93 1227-40.

Toggweiler J R, Dixon K and Bryan K 1989 Simulations of radiocarbon in a coarse-resolution world ocean model, 1, steady state prebomb distributions J. Geophys. Res. 94 8217-42 
Treguier A M and McWilliams J C 1990 Topographic influences on wind-driven, stratified flow in a beta-plane channel: An idealized model for the Antarctic Circumpolar Current J. Phys. Oceanogr. 20 321-43

Walker G T 1928 World weather, III Mem. R. Meteorol. Soc. 2 97-106

Weaver A J and Sarachik E S 1991 The role of mixed boundary conditions in numerical models of the ocean's climate J. Phys. Oceanogr. 21 1470-93

Weaver A J, Sarachik E J and Marotze J 1991 Freshwater fux forcing of decadal and interdecadal oceanic variability Nature $353836-8$

Welander P 1986 Thermohaline effects in the ocean circulation and related simple models Large-scale transport processes in oceans and atmosphere J Willebrand and DLT Anderson (Dordrecht: Reidel) pp 163-200

Wolff J O, Maier-Reimer E and Olbers D J 1991 Wind-driven flow over topography in a zonal beta-plane channel: A quasi-geostrophic model of the Antarctic Circumpolar Current J. Phys. Oceanogr. 21 236-64

Wyrtki K 1985 Water displacements in the Pacific and the genesis of El Niño cycles J. Geophys. Res. 90 7129-32 